\title{
KOLMOGOROV-TYPE THEORY OF COMPRESSIBLE TURBULENCE AND INVISCID LIMIT OF THE NAVIER-STOKES EQUATIONS IN $\mathbb{R}^{3}$
}

\author{
GUI-QIANG G. CHEN AND JAMES GLIMM
}

\begin{abstract}
We are concerned with the inviscid limit of the Navier-Stokes equations to the Euler equations for compressible fluids in $\mathbb{R}^{3}$. Motivated by the Kolmogorov hypothesis (1941) for incompressible flow, we introduce a Kolmogorov-type hypothesis for barotropic flows, in which the density and the sonic speed normally vary significantly. We then observe that the compressible Kolmogorov-type hypothesis implies the uniform boundedness of some fractional derivatives of the weighted velocity and sonic speed in the space variables in $L^{2}$, which is independent of the viscosity coefficient $\mu>0$. It is shown that this key observation yields the equicontinuity in both space and time of the density in $L^{\gamma}$ and the momentum in $L^{2}$, as well as the uniform bound of the density in $L^{q_{1}}$ and the velocity in $L^{q_{2}}$ independent of $\mu>0$, for some fixed $q_{1}>\gamma$ and $q_{2}>2$, where $\gamma>1$ is the adiabatic exponent. These results lead to the strong convergence of solutions of the Navier-Stokes equations to a solution of the Euler equations for barotropic fluids in $\mathbb{R}^{3}$. Not only do we offer a framework for mathematical existence theories, but also we offer a framework for the interpretation of numerical solutions through the identification of a function space in which convergence should take place, with the bounds that are independent of $\mu>0$, that is in the high Reynolds number limit.
\end{abstract}

\section{INTRODUCTION}

The purpose of this paper is to establish a framework for the existence theory for the Euler equations for compressible fluids. Motivated by a physically well accepted hypothesis by Kolmogorov [25, 26], Assumption (K41), for incompressible flow, we introduce a corresponding compressible Kolmogorov-type hypothesis (CKH) for barotropic flows, where the density and the sonic speed normally vary significantly, which play an essential role in compressible turbulence. The K41 scaling laws are derived from a dimensional analysis which pertains to the Euler equations; see [1].

A further contribution of this paper is that a weaker version Assumption (CKHw) of Assumption $(\mathrm{CKH})$ is sufficient to establish our main result that the convergence of weak solutions of the Navier-Stokes equations (in the sense of Definition 1.1 as introduced below) through a subsequence to a solution of the Euler equations is guaranteed. In particular, Assumption ( $\mathrm{CKHw})$, combined with the uniform energy bound, for the weak solutions of the Navier-Stokes equations with finite-energy initial data yields some uniform high integrability of the weak solutions with respect to the viscosity coefficients (i.e., the high Reynolds number).

Date: June 11, 2019.

2010 Mathematics Subject Classification. 35Q30, 76N10, 76F02, 76N17, 65M12, 35L65.

Key words and phrases. Inviscid limit, convergence, compressible fluids, Navier-Stokes equations, vanishing viscosity, compressible Kolmogorov-type hypotheses, turbulence, equicontinuity, existence, weak solutions, Euler equations, numerical convergence. 
As is well-known, there are two types of turbulence: driven turbulence by a forcing function and transient turbulence by (strong) initial conditions. The same flow (in a turbulent wind tunnel or turbulent flow in a pipe) could be of either type depending on how the system is modeled: If the pipe is considered in isolation, the flow might be forced; if the force is a flow connection from a reservoir to the wind tunnel or pipe, and the reservoir is part of the model, then the turbulence arises from initial conditions, and the turbulence will die out when the reservoir is exhausted and no longer drives the flow. Thus, the distinction between the two (driven turbulence and transient) is to some extent a matter of points of view and of modeling convenience. In this paper, our framework is focused on transient turbulence, though the forced turbulence is also included. For some recent developments in the mathematical study of energy dissipation in body-forced turbulence for incompressible flow, see Constantin-Doering [9], Doering-Foias [13], and the references cited therein.

Our main result is to address the issue of function spaces and norms in which the convergence might occur. The rate of dissipation of kinetic energy, $\epsilon$, in the sense of fully developed turbulence, i.e., in the present context, for the Euler equations, is understood as the cascade of transfer of energy to progressively smaller scales, according to the K41 theory. In a NavierStokes context, this dissipation cascade terminates with the dissipation of kinetic energy into heat, i.e., molecular motions. The question remains as to in what norm or function space to express this dissipation cascade property and convergence results, and in which the convergence of statistical ensemble occurs. Our main result addresses the question of function spaces and norms, which is developed from our previous work on the incompressible flow in [3] while the distinction among time, space, space-time, and ensemble averages relates to the well-known ergodic hypothesis and is out of the scope of the present paper.

We refer to [46] for a theoretical analysis of scaling laws for fully developed compressible fluid turbulence and to [49] for highly resolved numerical simulations of compressible turbulence. We do not attempt to survey the large literature on this topic, but additional references can be traced from these two. The central complication in passing from incompressible to compressible turbulence is the presence of additional dimensionless variables, so that scaling relations, rather than yielding a closed form expression for the decay of the velocity spectrum, as in Assumption (K41), now possess an additional dimensionless expression to be resolved by experiment or simulation. This additional variable enters in the prefactor, $\epsilon^{\frac{2}{3}}$ in (K41), and does not affect the exponent $-\frac{5}{3}$. Additionally, there are a variety of choices for the spectrum to be analyzed. Common choices are $\mathbf{u}, \sqrt{\rho} \mathbf{u}$, and $\sqrt[3]{\rho} \mathbf{u}$. The scaling exponent in these studies is never larger than $-\frac{5}{3}$, and is usually smaller. The choice $\sqrt[3]{\rho} \mathbf{u}$ is motivated by studies of high order structure functions.

Mathematically, our result has the status of an informed conjecture and mathematically rigorous consequences of this conjecture. Numerical analysts may find the framework useful, in view of the many difficulties involved in assessing the convergence of numerical simulations of turbulent and turbulent mixing flows. We expect that many physicists will accept the conclusions as being correct, even if unproven mathematically. There has been some discussion regarding the Kolmogorov exponent $\frac{5}{3}$ which occurs in Assumption $(\mathrm{CKH})$. We note that the main results (if not the detailed estimates) are not sensitive to this specific number, and corrections (as conventionally understood) to it due to intermittency do not affect our result. In fact, our rigorous argument works for an even weaker version, Assumption (CKHw). Most physicists would accept the stronger hypothesis, which we label (CKHi) $(i$ denotes intermittency), in which the assumed exponent is larger than $\frac{5}{3}$. This distinction has an important 
consequence as discussed in Section 6 that, for (CKHi) for incompressible flow, the energy is constant in time.

Not only do we offer a framework for mathematical existence theories, but also we offer a framework for the interpretation of numerical solutions of the Navier-Stokes equations. Only for very modest problems and with the largest computers can converged solutions of the Navier-Stokes equations be achieved. These solutions are called direct numerical solutions (DNS). For most solutions of interest to science or engineering, the large eddy simulations (LES) or Reynolds Averaged Navier-Stokes (RANS) simulations are required. We discuss here the more accurate LES methodology. Briefly, it employs a numerical grid which will resolve some but not all of the turbulent eddies. The smallest of those, below the level of the grid spacing, are not resolved. However, either (a) the Navier-Stokes equations are modified with additional subgrid scale (SGS) terms to model the influence of the unresolved scales on those that are being computed or (b) the numerical algorithm is modified in some manner to accomplish this effect in some other way. The present article contributes to this analysis through the introduction of a function space in which convergence should take place, with bounds that are independent of the viscosity coefficients, that is in the high Reynolds number limit.

Because of the common occurrence of high Reynolds numbers in flows of practical and scientific interest and the need to perform LES simulations to achieve scientific understanding and engineering designs, we observe that the existence theories for the Euler equations are relevant to the mathematical theories of numerical analysis.

We further remark that, for incompressible flow, Onsager's conjecture [43] states that weak solutions of the Euler equations for incompressible fluids in $\mathbb{R}^{3}$ conserve energy only if they have a certain minimal smoothness of the order of $\frac{1}{3}$-fractional derivatives in $\mathbf{x} \in \mathbb{R}^{3}$ and that they dissipate energy if they are rougher (also see [4]). For barotropic flows, the appearance of shock waves makes the energy always dissipative, so weak solutions of the Euler equations for compressible fluids in $\mathbb{R}^{3}$ do not conserve energy in general.

More precisely, consider the following Navier-Stokes equations for compressible fluids in $\mathbb{R}^{3}$ :

$$
\left\{\begin{array}{l}
\partial_{t} \rho+\nabla \cdot(\rho \mathbf{u})=0, \\
\partial_{t}(\rho \mathbf{u})+\nabla \cdot(\rho \mathbf{u} \otimes \mathbf{u})+\nabla p=\nabla \cdot \Sigma+\rho \mathbf{f},
\end{array}\right.
$$

with Cauchy data:

$$
\left.(\rho, \mathbf{u})\right|_{t=0}=\left(\rho_{0}(\mathbf{x}), \mathbf{u}_{0}(\mathbf{x})\right),
$$

where $\rho$ is the fluid density, $\mathbf{u}$ the velocity, $p$ the pressure, $\Sigma=\Sigma(\nabla \mathbf{u})$ the deviatoric stress tensor, $\nabla$ the gradient with respect to the space variable $\mathbf{x} \in \mathbb{R}^{3}, \mathbf{u} \otimes \mathbf{u}=\left(u_{i} u_{j}\right)$ the $3 \times 3$ matrix for $\mathbf{u}=\left(u_{1}, u_{2}, u_{3}\right)$, and $\mathbf{f}=\mathbf{f}(t, \mathbf{x})$ a given external force. The general form of the stress tenor $\Sigma=\Sigma(\nabla \mathbf{u})$ is

$$
\Sigma(\nabla \mathbf{u}):=2 \mu D(\nabla \mathbf{u})+\lambda(\nabla \cdot \mathbf{u}) \mathbf{I}_{3 \times 3} \quad \text { with } D(\nabla \mathbf{u}):=\frac{1}{2}\left(\nabla \mathbf{u}+(\nabla \mathbf{u})^{\top}\right),
$$

where $\max \{\mu,|\lambda|\} \in\left(0, \mu_{0}\right)$ for some $\mu_{0}>0, \lambda+\frac{2}{3} \mu \geq 0, \lambda=\lambda(\mu) \rightarrow 0$ as $\mu \rightarrow 0$, and $\mathbf{I}_{3 \times 3}$ is the $3 \times 3$ identity matrix. Mathematically, it suffices to require that $\lambda+2 \mu>0$ with $\lambda=\lambda(\mu) \rightarrow 0$ as $\mu \rightarrow 0$.

For ideal barotropic fluids, the pressure-density relation is

$$
p=p(\rho):=\kappa \rho^{\gamma},
$$


where $\gamma>1$ is the adiabatic exponent and $\kappa>0$ is a constant, and the internal energy is

$$
e=\frac{p}{(\gamma-1) \rho}=\frac{c^{2}}{\gamma-1}
$$

where $c$ is the sonic speed. Then the total energy is

$$
E=\frac{1}{2} \rho|\mathbf{u}|^{2}+\rho e=\rho\left(\frac{1}{2}|\mathbf{u}|^{2}+\frac{c^{2}}{\gamma-1}\right)=\frac{1}{2}|\sqrt{\rho} \mathbf{u}|^{2}+\frac{1}{\gamma-1}(\sqrt{\rho} c)^{2} .
$$

For clarity of presentation, we focus on periodic solutions with period $\mathbb{T}_{P}=\left[-\frac{P}{2}, \frac{P}{2}\right]^{3} \subset$ $\mathbb{R}^{3}, P>0$; that is,

$$
\left(\rho^{\mu}, \mathbf{u}^{\mu}\right)\left(t, \mathbf{x}+P \mathbf{e}_{i}\right)=\left(\rho^{\mu}, \mathbf{u}^{\mu}\right)(t, \mathbf{x})
$$

with $\left\{\mathbf{e}_{i}\right\}_{i=1}^{3}$ the canonic basis in $\mathbb{R}^{3}$. Other cases can be analyzed correspondingly.

Throughout this paper, we always assume that $\mathbf{f} \in L_{\text {loc }}^{1}\left(\mathbb{R}_{+} ; L^{\frac{2 \gamma}{\gamma-1}}\left(\mathbb{T}_{P}\right)\right)$ is periodic in $\mathbf{x}$ with period $\mathbb{T}_{P}$. Assume that $\left(\rho_{0}, \mathbf{u}_{0}\right)$ are periodic in $\mathbf{x} \in \mathbb{R}^{3}$ with period $\mathbb{T}_{P}$ so that

$$
\begin{aligned}
& \rho_{0} \neq 0, \quad \rho_{0} \geq 0 \text { a.e. in } \Omega, \\
& \mathbf{m}_{0}=\rho_{0} \mathbf{u}_{0} \in L^{\frac{2 \gamma}{\gamma+1}},
\end{aligned}
$$

and that the initial total energy is finite in $\mathbb{T}_{P}$ :

$$
\mathcal{E}_{0}=\int_{\mathbb{T}_{P}}\left(\frac{1}{2} \rho_{0}\left|\mathbf{u}_{0}\right|^{2}+\frac{p\left(\rho_{0}\right)}{\gamma-1}\right) \mathrm{d} \mathbf{x}<\infty
$$

In this paper, we focus on the following class of periodic weak solutions $\left(\rho^{\mu}, \mathbf{u}^{\mu}\right)=$ $\left(\rho^{\mu}, \mathbf{u}^{\mu}\right)(t, \mathbf{x})$ with period $\mathbb{T}_{P}$ of the Cauchy problem (1.1)-(1.2).

Definition 1.1 (Weak solutions of the Navier-Stokes equations). A periodic vector function $\left(\rho^{\mu}, \mathbf{u}^{\mu}\right)=\left(\rho^{\mu}, \mathbf{u}^{\mu}\right)(t, \mathbf{x})$ with period $\mathbb{T}_{P}$ is a weak solution of the Cauchy problem (1.1)-(1.2), provided that $\left(\rho^{\mu}, \mathbf{u}^{\mu}\right)$ satisfies the following:

(i) For any $T>0,\left(\rho^{\mu}, \mathbf{u}^{\mu}\right)$ satisfies the equations in (1.1) in the sense of distributions in $\mathbb{R}_{T}^{3}=[0, T) \times \mathbb{R}^{3}$, the initial condition (1.2), and the following properties:

$$
\begin{aligned}
& \mathbf{u}^{\mu} \in L^{2}\left(0, T ; H^{1}\left(\mathbb{T}_{P}\right)\right), \quad \nabla \mathbf{u}^{\mu} \in L^{2}\left([0, T) \times \mathbb{T}_{P}\right), \\
& \rho^{\mu} \in C\left([0, T] ; L_{\mathrm{w}}^{\gamma}\left(\mathbb{T}_{P}\right)\right) \cap L^{p}\left([0, T) \times \mathbb{T}_{P}\right) \quad \text { for } 1 \leq p \leq \max \left\{\frac{5 \gamma-3}{3}, 1\right\}, \\
& \mathbf{m}^{\mu}:=\rho^{\mu} \mathbf{u}^{\mu} \in C\left([0, T] ; L_{\mathrm{w}}^{\frac{2 \gamma}{\gamma+1}}\left(\mathbb{T}_{P}\right)\right), \quad \rho^{\mu}\left|\mathbf{u}^{\mu}\right|^{2} \in L^{\infty}\left(0, T ; L^{1}\left(\mathbb{T}_{P}\right)\right),
\end{aligned}
$$

where a vector $\mathbf{v} \in C\left([0, T] ; L_{\mathrm{w}}^{q}\left(\mathbb{T}_{P}\right)\right)$ means that $\mathbf{v} \in L^{\infty}\left(0, T ; L^{q}\left(\mathbb{T}_{P}\right)\right)$ and $\mathbf{v}$ is continuous in $t$ with values in $L^{q}\left(\mathbb{T}_{P}\right)$ endowed with the weak topology;

(ii) The following inequality holds in the sense of distributions in $\mathbb{R}_{T}^{3}$ :

$$
\partial_{t} E^{\mu}+\nabla \cdot\left(\left(E^{\mu}+p^{\mu}\right) \mathbf{u}^{\mu}\right)+\mu\left|\nabla \mathbf{u}^{\mu}\right|^{2}+(\lambda+\mu)\left|\nabla \cdot \mathbf{u}^{\mu}\right|^{2} \leq \nabla \cdot\left(\Sigma^{\mu}\left(\nabla \mathbf{u}^{\mu}\right) \mathbf{u}^{\mu}\right)+\mathbf{m}^{\mu} \cdot \mathbf{f} .
$$

The global existence of weak solutions of the Cauchy problem (1.1)-(1.2) in the sense of Definition 1.1 was first established by P.-L. Lions [36] for $\gamma>\frac{9}{5}$, and was extended by Feireisl-Novotny-Petzeltova [16] for $\gamma \in\left(\frac{3}{2}, \frac{9}{5}\right]$. Some further a priori estimates and properties of solutions of the Navier-Stokes equations (1.1) for compressible fluids can be found in [17, 36 , and the references cited therein. Thus, throughout the paper, we assume either $\gamma>\frac{3}{2}$ or the existence of weak solutions satisfying (i)-(ii) and $\gamma>1$. 
The energy inequality (1.11) implies that

$$
\begin{aligned}
& \int_{\mathbb{T}_{P}}\left(\frac{1}{2}\left|\sqrt{\rho^{\mu}} \mathbf{u}^{\mu}\right|^{2}+\frac{1}{\gamma-1}\left(\sqrt{\rho^{\mu}} c^{\mu}\right)^{2}\right)(t, \mathbf{x}) \mathrm{d} \mathbf{x}+\int_{0}^{\mathrm{t}} \int_{\mathbb{T}_{P}}\left(\mu\left|\nabla \mathbf{u}^{\mu}\right|^{2}+(\lambda+\mu)\left|\nabla \cdot \mathbf{u}^{\mu}\right|^{2}\right) \mathrm{d} \mathbf{x} \mathrm{dt} \\
& \leq \mathcal{E}_{0}+\int_{0}^{t} \int_{\mathbb{T}_{P}} \mathbf{m}^{\mu} \cdot \mathbf{f} \mathrm{d} \mathbf{x} \mathrm{dt}<\infty
\end{aligned}
$$

where $\mathcal{E}_{0}$ is the initial energy over period $\mathbb{T}_{P}$, independent of the viscosity coefficient $\mu$ :

$$
\mathcal{E}_{0}:=\int_{\mathbb{T}_{P}}\left(\frac{1}{2}\left|\sqrt{\rho_{0}} \mathbf{u}_{0}\right|^{2}+\frac{1}{\gamma-1}\left(\sqrt{\rho_{0}} c_{0}\right)^{2}\right)(t, \mathbf{x}) \mathrm{d} \mathbf{x}
$$

Notice that there exists $C_{\gamma}>0$ depending only on $\gamma$ such that

$$
\left|\mathbf{m}^{\mu} \cdot \mathbf{f}\right| \leq \frac{1}{2}\left|\sqrt{\rho^{\mu}} \mathbf{u}^{\mu}\right|^{2}+\frac{1}{\gamma-1}\left(\sqrt{\rho^{\mu}} c^{\mu}\right)^{2}+C_{\gamma}|\mathbf{f}|^{\frac{2 \gamma}{\gamma-1}} .
$$

Then, for any $T>0$, we use the Gronwall inequality to conclude that there exists a constant $M_{T}$ that is independent of the viscosity coefficient $\mu$, but may depend on $T, \gamma>1$, $\mathcal{E}_{0}$, and $\|\mathbf{f}\|_{L^{1}\left(0, T ; L^{\frac{2 \gamma}{\gamma-1}}\left(\mathbb{T}_{P}\right)\right)}$, such that

$$
\begin{aligned}
& \int_{\mathbb{T}_{P}}\left(\frac{1}{2}\left|\sqrt{\rho^{\mu}} \mathbf{u}^{\mu}\right|^{2}+\frac{1}{\gamma-1}\left(\sqrt{\rho^{\mu}} c^{\mu}\right)^{2}\right)(t, \mathbf{x}) \mathrm{d} \mathbf{x}+\int_{0}^{\mathrm{t}} \int_{\mathbb{T}_{\mathrm{P}}}\left(\mu\left|\nabla \mathbf{u}^{\mu}\right|^{2}+(\lambda+\mu)\left|\nabla \cdot \mathbf{u}^{\mu}\right|^{2}\right) \mathrm{d} \mathbf{x} \mathrm{dt} \\
& \leq M_{T}<\infty .
\end{aligned}
$$

From now on, we always denote $M_{T}$ as a universal constant, independent of the viscosity coefficient $\mu>0$, but may depend on $T, \gamma>1, \mathcal{E}_{0}$, and $\|\mathbf{f}\|_{L^{1}\left(0, T ; L^{\frac{2 \gamma}{\gamma-1}}\left(\mathbb{T}_{P}\right)\right)}$.

In this paper, we consider all the weak solutions $\left(\rho^{\mu}, \mathbf{u}^{\mu}\right)$ of the Navier-Stokes equations (1.1) with initial condition (1.2) in the sense of Definition 1.1 for all $\gamma>1$ and study their strong convergence to a weak solution $(\rho, \mathbf{u})$ of the following Euler equations for barotropic compressible fluids in $\mathbb{R}^{3}$ :

$$
\left\{\begin{array}{l}
\partial_{t} \rho+\nabla \cdot(\rho \mathbf{u})=0 \\
\partial_{t}(\rho \mathbf{u})+\nabla \cdot(\rho \mathbf{u} \otimes \mathbf{u})+\nabla p=\rho \mathbf{f}
\end{array}\right.
$$

with the same Cauchy data (1.2) in the following sense:

Definition 1.2 (Weak solutions of the Euler equations). A periodic vector function $(\rho, \mathbf{m})=$ $(\rho, \rho \mathbf{u})(t, \mathbf{x})$ with period $\mathbb{T}_{P}$ is a weak solution of the Cauchy problem (1.2) and (1.14), provided that $(\rho, \mathbf{m})$ satisfies the following:

(i) For any $\varphi \in C_{0}^{\infty}\left(\mathbb{R}_{+} \times \mathbb{R}^{3} ; \mathbb{R}\right)$,

$$
\int_{0}^{\infty} \int_{\mathbb{R}^{3}}\left(\rho \varphi_{t}+\mathbf{m} \cdot \nabla \varphi\right) \mathrm{d} \mathbf{x} \mathrm{dt}+\int_{\mathbb{R}^{3}} \rho_{0}(\mathbf{x}) \varphi(0, \mathbf{x}) \mathrm{d} \mathbf{x}=0
$$

(ii) For any $\varphi \in C_{0}^{\infty}\left(\mathbb{R}_{+} \times \mathbb{R}^{3} ; \mathbb{R}^{3}\right)$,

$$
\begin{aligned}
& \int_{0}^{\infty} \int_{\mathbb{R}^{3}}\left(\mathbf{m} \cdot \boldsymbol{\varphi}_{t}+\frac{\mathbf{m} \otimes \mathbf{m}}{\rho}: \nabla \boldsymbol{\varphi}+p(\rho) \operatorname{div} \varphi+\rho \mathbf{f} \cdot \boldsymbol{\varphi}\right) \mathrm{d} \mathbf{x d t} \\
& +\int_{\mathbb{R}^{3}} \mathbf{m}_{0}(\mathbf{x}) \cdot \boldsymbol{\varphi}(0, \mathbf{x}) \mathrm{d} \mathbf{x}=0
\end{aligned}
$$

where $A: B$ is the matrix product $\sum_{i, j} a_{i j} b_{i j}$ for $A=\left(a_{i j}\right)$ and $B=\left(b_{i j}\right)$; 
(iii) For all $t \in(0, \infty)$,

$$
\int_{\mathbb{T}_{P}} E(t, \mathbf{x}) \mathrm{d} \mathbf{x} \leq \int_{\mathbb{T}_{\mathrm{P}}} \mathrm{E}_{0}(\mathbf{x}) \mathrm{d} \mathbf{x}+\int_{0}^{\mathrm{t}} \int_{\mathbb{T}_{\mathrm{P}}} \mathbf{m}(\mathrm{s}, \mathbf{x}) \cdot \mathbf{f}(\mathrm{s}, \mathbf{x}) \mathrm{d} \mathbf{x} \mathrm{ds} .
$$

By contrast, less is known regarding the existence theory for the Euler equations (1.14) with the Cauchy data (1.2) even in the weak sense of Definition 1.2. For the compressible case, the analysis of [21, 40] gives weakly convergent subsequences, but the limit is not shown to satisfy the original equations, in that the interchange of limits with nonlinear terms in the equations is not justified in this analysis. On this basis, we state that the existence of solutions of the Cauchy problem (1.2) for the Euler equations (1.14) in $\mathbb{R}^{3}$ is open as is the convergence of the inviscid limit from the Navier-Stokes to the Euler equations. On the other hand, the Euler equations are fundamental for turbulence.

\section{The Kolmogorov-Type Hypothesis for Compressible Turbulence}

From the energy estimate (1.13) of weak solutions $\left(\rho^{\mu}, \mathbf{u}^{\mu}\right)$ of the Navier-Stokes equations (1.1) with finite-energy initial data (1.2) in the sense of Definition 1.1, for any $T>0$, there exists $M_{T}>0$ independent of $\mu$ such that

$$
\left\|\left(\sqrt{\rho^{\mu}} \mathbf{u}^{\mu}, \sqrt{\rho^{\mu}} c\left(\rho^{\mu}\right)\right)\right\|_{L^{\infty}\left(0, T ; L^{2}\left(\mathbb{T}_{P}\right)\right)}^{2}+\sqrt{\mu}\left\|\nabla \mathbf{u}^{\mu}\right\|_{L^{2}\left([0, T) \times \mathbb{T}_{P}\right)} \leq M_{T}<\infty .
$$

From now on, $M_{T}$ is always denoted as a universal constant, independent of $\mu>0$, but may depend on $T, \gamma>1, \mathcal{E}_{0}$, and $\|f\|_{L^{1}\left(0, T ; L^{\frac{2 \gamma}{\gamma-1}}\right.}\left(\mathbb{T}_{P}\right)$.

Then the total energy $\mathcal{E}(t)$ per unit mass at time $t$ for isotropic turbulence is:

$$
\begin{aligned}
\mathcal{E}(t) & =\frac{1}{\left|\mathbb{T}_{P}\right|} \int_{\mathbb{T}_{P}}\left(\frac{1}{2}\left|\sqrt{\rho^{\mu}} \mathbf{u}^{\mu}\right|^{2}+\frac{1}{\gamma-1}\left(\sqrt{\rho^{\mu}} c\left(\rho^{\mu}\right)\right)^{2}\right) \mathrm{d} \mathbf{x} \\
& =\sum_{k \geq 0} \mathbb{E}(t, k)=\sum_{k \geq 0} 4 \pi q(t, k) k^{2} .
\end{aligned}
$$

Here $\mathbb{E}(t, k), k=|\mathbf{k}|$, is the energy wavenumber spectrum, $q(t, k)$ can be interpreted as the density of contributions in wavenumber space to the total energy, which is sometimes called the spectral density, and $\mathbf{k}=\left(k_{1}, k_{2}, k_{3}\right)=\frac{2 \pi}{P}\left(n_{1}, n_{2}, n_{3}\right) \in \mathbb{R}^{3}$, with $n_{j}=0, \pm 1, \pm 2, \cdots$, and $j=1,2,3$, is the discrete wavevector in the Fourier transform:

$$
\widehat{(\sqrt{\rho} \mathbf{u}}, \widehat{\sqrt{\rho} c(\rho)})(t, \mathbf{k})=\frac{1}{\left|\mathbb{T}_{P}\right|} \int_{\mathbb{T}_{P}}(\sqrt{\rho} \mathbf{u}, \sqrt{\rho} c(\rho))(t, \mathbf{x}) e^{-i \mathbf{k} \cdot \mathbf{x}} \mathrm{d} \mathbf{x}
$$

of the weighted velocity and sonic speed $(\sqrt{\rho} \mathbf{u}, \sqrt{\rho} c(\rho))(t, \mathbf{x})$ in the $\mathbf{x}$-variable. Then

$$
(\sqrt{\rho} \mathbf{u}, \sqrt{\rho} c(\rho))(t, \mathbf{x})=\sum_{\mathbf{k}}(\widehat{\sqrt{\rho} \mathbf{u}}, \widehat{\sqrt{\rho} c(\rho)})(t, \mathbf{k}) e^{i \mathbf{k} \cdot \mathbf{x}}
$$

We adopt Kolmogorov's assumptions (1941) in his description of isotropic incompressible turbulence in Kolmogorov [25, 26] (also see McComb [38]) to introduce the following compressible Kolmogorov-type hypothesis $(\mathrm{CKH})$ in mathematical terms: 
Assumption (CKH): For any $T>0$, there exist $M_{T}>0$ and $k_{*}$ (sufficiently large) independent of the viscosity $\mu$, which may depend on $T, \mathcal{E}_{0}, \gamma>1$, and $\|\mathbf{f}\|_{L^{1}\left(0, T ; L^{\frac{2 \gamma}{\gamma-1}}\left(\mathbb{T}_{P}\right)\right)}$, such that, for $k=|\mathbf{k}| \geq k_{*}$,

$$
\int_{0}^{T} \mathbb{E}(t, k) \mathrm{dt} \leq \mathrm{M}_{\mathrm{T}} \mathrm{k}^{-\frac{5}{3}}
$$

For general turbulence, the energy wavenumber spectrum $\mathbb{E}(t, k)$ in (2.2) may be replaced by $\mathbb{E}(t, k, \phi, \theta)$ in the spherical coordinates $(k, \phi, \theta), 0 \leq \phi \leq \pi, 0 \leq \theta \leq 2 \pi$, in the $\mathbf{k}$-space, but it should be in the same asymptotics as in (2.2) for sufficiently high wavenumber $k=|\mathbf{k}|$.

For our analysis, the following weaker version of Assumption (CKHw) is sufficient:

Assumption (CKHw): For any $T>0$, there exist $M_{T}=C_{T}>0$ and $k_{*}$ (sufficiently large) independent of the viscosity coefficient $\mu$ such that, for $k=|\mathbf{k}| \geq k_{*}$,

$$
\left.\left.\sup _{k \geq k_{*}}\left(|\mathbf{k}|^{3+\beta} \int_{0}^{T} \mid \widehat{(\sqrt{\rho} \mathbf{u}}, \widehat{\sqrt{\rho} c(\rho)}\right)(t, \mathbf{k})\right|^{2} \mathrm{dt}\right) \leq \mathrm{M}_{\mathrm{T}} \quad \text { for some } \beta>0 .
$$

In Assumption (CKHw), the case $\beta=\frac{2}{3}$ in (2.5) corresponds to Assumption ( $\left.\mathrm{CKH}\right)$ in (2.4). Assumption (CKHi) corresponds the requirement that $\beta>\frac{2}{3}$.

As indicated in Chen-Glimm [3], a mathematical proof of Assumption (CKH) may well depend on developing a mathematical version of the renormalization group, which has proved to be very powerful in theoretical physics calculations.

\section{3. $L^{2}$-Equicontinuity of the Weighted Velocity and Sonic Speed in the Space VARIABLES, INDEPENDENT OF THE VISCOSITY}

In this section, we show that the compressible Kolmogorov-type hypothesis, Assumption (CKHw), implies a uniform bound of the weighted velocity $\left(\sqrt{\rho^{\mu}} \mathbf{u}^{\mu}\right)(t, \mathbf{x})$ and sonic speed $\left(\sqrt{\rho^{\mu}} c\left(\rho^{\mu}\right)\right)(t, \mathbf{x})$ in $L^{2}\left(0, T ; H^{\alpha}\left(\mathbb{T}_{P}\right)\right)$ for any $\alpha \in\left(0, \frac{\beta}{2}\right)$, especially the uniform equicontinuity of the weighted velocity $\left(\sqrt{\rho^{\mu}} \mathbf{u}^{\mu}\right)(t, \mathbf{x})$ and the weighted sonic speed $\left(\sqrt{\rho^{\mu}} c\left(\rho^{\mu}\right)\right)(t, \mathbf{x})$ in the space variables in $L^{2}\left([0, T) \times \mathbb{T}_{P}\right)$, independent of $\mu>0$. This also implies the uniform equicontinuity of the density and momentum in appropriate norms as specified below.

Proposition 3.1. Under Assumption (CKHw), for any $T \in(0, \infty)$, there exists $M_{T}>0$, independent of $\mu>0$, such that

$$
\left\|\left(\sqrt{\rho^{\mu}} \mathbf{u}^{\mu}, \sqrt{\rho^{\mu}} c\left(\rho^{\mu}\right)\right)\right\|_{L^{2}\left(0, T ; H^{\alpha}\left(\mathbb{T}_{P}\right)\right)} \leq M_{T}<\infty,
$$

where $\alpha \in\left(0, \frac{\beta}{2}\right)$. 
Proof. Set $\mathbf{w}^{\mu}:=\left(\sqrt{\rho^{\mu}} \mathbf{u}^{\mu}, \sqrt{\rho^{\mu}} c\left(\rho^{\mu}\right)\right)$. Using the definition of fractional derivatives via the Fourier transform, the Parseval identity, and Assumption (CKHw), i.e., (2.4), we have

$$
\begin{aligned}
& \int_{0}^{T} \int_{\mathbb{T}_{P}}\left|D_{\mathbf{x}}^{\alpha} \mathbf{w}^{\mu}(t, \mathbf{x})\right|^{2} \mathrm{~d} \mathbf{x} \mathrm{dt} \\
& \leq M_{1} \int_{0}^{T}\left(\sum_{\mathbf{k}}|\mathbf{k}|^{2 \alpha}\left|\widehat{\mathbf{w}^{\mu}}(t, \mathbf{k})\right|^{2}\right) \mathrm{dt} \\
& =M_{1} \int_{0}^{T}\left(\sum_{0 \leq|\mathbf{k}| \leq k_{*}}|\mathbf{k}|^{2 \alpha}\left|\widehat{\mathbf{w}^{\mu}}(t, \mathbf{k})\right|^{2}\right) \mathrm{dt}+\mathrm{M}_{1} \int_{0}^{\mathrm{T}}\left(\sum_{|\mathbf{k}|>\mathrm{k}_{*}}|\mathbf{k}|^{2 \alpha}\left|\widehat{\mathbf{w}^{\mu}}(\mathrm{t}, \mathbf{k})\right|^{2}\right) \mathrm{dt} \\
& \leq M_{1} k_{*}^{2 \alpha} \int_{0}^{T}\left(\sum_{0 \leq|\mathbf{k}| \leq k_{*}}\left|\widehat{\mathbf{w}^{\mu}}(t, \mathbf{k})\right|^{2}\right) \mathrm{dt}+\mathrm{M}_{2} \sum_{|\mathbf{k}| \geq \mathrm{k}_{*}}|\mathbf{k}|^{2 \alpha-3-\beta} \\
& \leq M_{1} k_{*}^{2 \alpha} \int_{0}^{T}\left(\sum_{\mathbf{k}}\left|\widehat{\mathbf{w}^{\mu}}(t, \mathbf{k})\right|^{2}\right) \mathrm{dt}+\mathrm{M}_{3} \sum_{\mathrm{k} \geq \mathrm{k}_{*}} \mathrm{k}^{2 \alpha-1-\beta} \\
& \leq M_{1} k_{*}^{2 \alpha} \int_{0}^{T} \int_{\mathbb{T}_{P}}\left|\mathbf{w}^{\mu}(t, \mathbf{x})\right|^{2} \mathrm{~d} \mathbf{x} \mathrm{dt}+\mathrm{M}_{4} \mathrm{k}_{*}^{2 \alpha-\beta} \\
& \leq M_{5} k_{*}^{2 \alpha} T\left(\int_{\mathbb{T}_{P}}\left|\mathbf{w}_{0}(\mathbf{x})\right|^{2} \mathrm{~d} \mathbf{x}+\int_{0}^{T} \int_{\mathbb{T}_{P}}|\mathbf{f}(\mathrm{t}, \mathbf{x})|^{2} \mathrm{~d} \mathbf{x} \mathrm{dt}\right)+\mathrm{M}_{4} \mathrm{k}_{*}^{2 \alpha-\beta} \\
& \leq M_{T}^{2}<\infty, \quad
\end{aligned}
$$

since $\alpha<\frac{\beta}{2}$, which $M_{j}, j=1, \cdots, 5$, and $M_{T}$ are the constants independent of $\mu$, but may depends on $T, k_{*}$, and $\alpha$. This completes the proof.

Proposition 3.1 directly yields the uniform equicontinuity of $\left(\sqrt{\rho^{\mu}} \mathbf{u}^{\mu}(t, \mathbf{x}), \sqrt{\rho^{\mu}} c\left(\rho^{\mu}\right)\right)$ in $\mathbf{x}$ in $L^{2}\left([0, T) \times \mathbb{T}_{P}\right)$ independent of $\mu>0$, which particularly implies the uniform equicontinuity of the density $\rho^{\mu}$ in both space and time in $L^{\gamma}$ and the momentum $\mathbf{m}^{\mu}$ in $L^{2}$, as well as the corresponding uniform high integrability of $\left(\sqrt{\rho^{\mu}} \mathbf{u}^{\mu}(t, \mathbf{x}), \sqrt{\rho^{\mu}} c\left(\rho^{\mu}\right)\right)$, independent of $\mu>0$.

Proposition 3.2. Under Assumption (CKHw), for any $T \in(0, \infty)$, there exists $M_{T}>0$, independent of $\mu>0$, such that

(i) There exists $q=q(\beta)>2$ so that

$$
\left\|\left(\sqrt{\rho^{\mu}} \mathbf{u}^{\mu}, \sqrt{\rho^{\mu}} c\left(\rho^{\mu}\right)\right)\right\|_{L^{q}\left([0, T] \times \mathbb{T}_{P}\right)} \leq M_{T}
$$

(ii) There exists $\alpha_{1} \in\left(0, \frac{\beta}{4}\right)$ so that

$$
\begin{aligned}
& \int_{0}^{T} \int_{\mathbb{T}_{P}}\left|\rho^{\mu}(t, \mathbf{x}+\Delta \mathbf{x})-\rho^{\mu}(t, \mathbf{x})\right|^{\gamma} \mathrm{d} \mathbf{x} \mathrm{dt} \\
& +\int_{0}^{T} \int_{\mathbb{T}_{P}}\left|\mathbf{m}^{\mu}(t, \mathbf{x}+\Delta \mathbf{x})-\mathbf{m}^{\mu}(t, \mathbf{x})\right|^{2} \mathrm{~d} \mathbf{x} \mathrm{dt} \leq \mathrm{M}_{\mathrm{T}}|\Delta \mathbf{x}|^{\alpha_{1}}
\end{aligned}
$$

In particular,

$$
\left\|\mathbf{m}^{\mu}\right\|_{L^{2}\left([0, T] \times \mathbb{T}_{P}\right)} \leq M_{T} .
$$

Proof. We divide the proof into three steps. 
1. The result in (i) directly follows by combining Proposition 3.1 with the Sobolev embedding theorem, since $\left(\sqrt{\rho^{\mu}} \mathbf{u}^{\mu}, \sqrt{\rho^{\mu}} c\left(\rho^{\mu}\right)\right)$ are uniformly bounded in $L^{2}\left(0, T ; H^{\alpha}\left(\mathbb{T}_{P}\right)\right)$ for $\alpha \in\left(0, \frac{\beta}{2}\right)$.

2. Proposition 3.1 implies that

$$
\int_{0}^{T} \int_{\mathbb{T}_{P}}\left|\left(\rho^{\mu}\right)^{\frac{\gamma}{2}}(t, \mathbf{x}+\Delta \mathbf{x})-\left(\rho^{\mu}\right)^{\frac{\gamma}{2}}(t, \mathbf{x})\right|^{2} \mathrm{~d} \mathbf{x d t} \leq \mathrm{M}_{\mathrm{T}}|\Delta \mathbf{x}|^{2 \alpha}
$$

When $\gamma \geq 2$, using that $|x-1|^{\gamma} \leq\left(x^{\frac{\gamma}{2}}-1\right)^{2}$ for any $x \geq 0$, we have

$$
\begin{aligned}
& \int_{0}^{T} \int_{\mathbb{T}_{P}}\left|\rho^{\mu}(t, \mathbf{x}+\Delta \mathbf{x})-\rho^{\mu}(t, \mathbf{x})\right|^{\gamma} \mathrm{d} \mathbf{x} \mathrm{dt} \\
& \leq \int_{0}^{T} \int_{\mathbb{T}_{P}}\left|\left(\rho^{\mu}\right)^{\frac{\gamma}{2}}(t, \mathbf{x}+\Delta \mathbf{x})-\left(\rho^{\mu}\right)^{\frac{\gamma}{2}}(t, \mathbf{x})\right|^{2} \mathrm{~d} \mathbf{x} \mathrm{dt} \leq \mathrm{M}_{\mathrm{T}}|\Delta \mathbf{x}|^{2 \alpha} .
\end{aligned}
$$

When $\gamma \leq 2$, since

$$
x-y=\left(x^{\frac{\gamma}{2}}\right)^{\frac{2}{\gamma}}-\left(y^{\frac{\gamma}{2}}\right)^{\frac{2}{\gamma}}=\frac{2}{\gamma} \int_{0}^{1}\left(\theta x^{\frac{\gamma}{2}}+(1-\theta) y^{\frac{\gamma}{2}}\right)^{\frac{2}{\gamma}-1} \mathrm{~d} \theta\left|\mathrm{x}^{\frac{\gamma}{2}}-\mathrm{y}^{\frac{\gamma}{2}}\right|
$$

for any $x>0$ and $y>0$, we have

$$
|x-y|^{\gamma} \leq C\left(x^{\frac{2-\gamma}{2}}+y^{\frac{2-\gamma}{2}}\right)\left|x^{\frac{\gamma}{2}}-y^{\frac{\gamma}{2}}\right|^{\gamma} .
$$

Then

$$
\begin{aligned}
& \quad \int_{0}^{T} \int_{\mathbb{T}_{P}}\left|\rho^{\mu}(t, \mathbf{x}+\Delta \mathbf{x})-\rho^{\mu}(t, \mathbf{x})\right|^{\gamma} \mathrm{d} \mathbf{x d t} \\
& \leq M_{T}\left(\int_{0}^{T} \int_{\mathbb{T}_{P}}\left(\left|\rho^{\mu}(t, \mathbf{x}+\Delta \mathbf{x})\right|+\left|\rho^{\mu}(t, \mathbf{x})\right|\right) \mathrm{d} \mathbf{x} \mathrm{dt}\right)^{\frac{2-\gamma}{2}} \\
& \quad \times\left(\int_{0}^{T} \int_{\mathbb{T}_{P}}\left|\left(\rho^{\mu}\right)^{\frac{\gamma}{2}}(t, \mathbf{x}+\Delta \mathbf{x})-\left(\rho^{\mu}\right)^{\frac{\gamma}{2}}(t, \mathbf{x})\right|^{2} \mathrm{~d} \mathbf{x d t}\right)^{\frac{\gamma}{2}} \\
& \leq M_{T}|\Delta \mathbf{x}|^{\alpha \gamma} .
\end{aligned}
$$

3. Proposition 3.1 and (3.5)-(3.6) imply

$$
\begin{aligned}
& \int_{0}^{T} \int_{\mathbb{T}_{P}}\left|\mathbf{m}^{\mu}(t, \mathbf{x}+\Delta \mathbf{x})-\mathbf{m}^{\mu}(t, \mathbf{x})\right|^{2} \mathrm{~d} \mathbf{x d t} \\
& \leq\left\|\sqrt{\rho^{\mu}}\right\|_{L^{2}\left(\mathbb{T}_{P}\right)}\left(\int_{0}^{T} \int_{\mathbb{T}_{P}}\left(\left|\left(\sqrt{\rho^{\mu}} \mathbf{u}^{\mu}\right)(t, \mathbf{x}+\Delta \mathbf{x})-\left(\sqrt{\rho^{\mu}} \mathbf{u}^{\mu}\right)(t, \mathbf{x})\right|^{2}\right) \mathrm{d} \mathbf{x d t}\right)^{\frac{1}{2}} \\
& \quad+\left\|\sqrt{\rho^{\mu}} \mathbf{u}^{\mu}\right\|_{L^{2}\left([0, T] \times \mathbb{T}_{P}\right)}\left(\int_{0}^{T} \int_{\mathbb{T}_{P}}\left(\left|\sqrt{\rho^{\mu}}(t, \mathbf{x}+\Delta \mathbf{x})-\sqrt{\rho^{\mu}}(t, \mathbf{x})\right|\right)^{2} \mathrm{~d} \mathbf{x d t}\right)^{\frac{1}{2}} \\
& \leq M_{T}|\Delta \mathbf{x}|^{\alpha}+M_{T}\left(\int_{0}^{T} \int_{\mathbb{T}_{P}}\left|\rho^{\mu}(t, \mathbf{x}+\Delta \mathbf{x})-\rho^{\mu}(t, \mathbf{x})\right|^{\gamma} \mathrm{d} \mathbf{x d t}\right)^{\frac{1}{2 \gamma}} \\
& \leq M_{T}\left(|\Delta \mathbf{x}|^{\alpha}+|\Delta \mathbf{x}|^{\alpha \min \left\{\frac{1}{\gamma}, \frac{1}{2}\right\}}\right) \\
& \leq M_{T}|\Delta \mathbf{x}|^{\alpha \min \left\{\frac{1}{\gamma}, \frac{1}{2}\right\}} .
\end{aligned}
$$

Combining (3.5)-(3.7) together and choosing $\alpha_{1}=\alpha \min \left\{\frac{1}{\gamma}, \frac{1}{2}\right\} \in\left(0, \frac{\beta}{4}\right)$ yield (3.3) . 
In particular,

$$
\int_{0}^{T} \int_{\mathbb{T}_{P}}\left|\mathbf{m}^{\mu}(t, \mathbf{x})\right|^{2} \mathrm{~d} \mathbf{x} \mathrm{dt} \leq\left\|\rho^{\mu}\right\|_{\mathrm{L}^{1}\left([0, \mathrm{~T}] \times \mathbb{T}_{\mathrm{P}}\right)}\left\|\sqrt{\rho^{\mu}} \mathbf{u}^{\mu}\right\|_{\mathrm{L}^{2}\left([0, \mathrm{~T}] \times \mathbb{T}_{\mathrm{P}}\right)} \leq \mathrm{M}_{\mathrm{T}}<\infty .
$$

This completes the proof.

\section{4. $L^{2}$-Equicontinuity of the Density and Momentum in the Time Variable, INDEPENDENT OF THE VISCOSITY}

In this section, we show that Proposition 3.1 implies the uniform equicontinuity of the density and momentum in the time variable $t>0$ in $L^{2}$, independent of $\mu>0$.

Proposition 4.1. For any $T>0$, there exist $\alpha_{2}=\alpha_{2}(\beta)>0$ depending only on $\beta$, and $M_{T}>0$ independent of $\mu>0$ such that, for all small $\triangle t>0$,

$$
\begin{aligned}
& \int_{0}^{T-\triangle t} \int_{\mathbb{T}_{P}}\left|\rho^{\mu}(t+\triangle t, \mathbf{x})-\rho^{\mu}(t, \mathbf{x})\right|^{\gamma} \mathrm{d} \mathbf{x d t} \\
& +\int_{0}^{T-\triangle t} \int_{\mathbb{T}_{P}}\left|\mathbf{m}^{\mu}(t+\triangle t, \mathbf{x})-\mathbf{m}^{\mu}(t, \mathbf{x})\right|^{2} \mathrm{~d} \mathbf{x} \mathrm{dt} \\
& \leq M_{T}(\triangle t)^{\alpha_{2}} \rightarrow 0 \quad \text { as } \triangle t \rightarrow 0 .
\end{aligned}
$$

Proof. For simplicity, we drop the superscript $\mu>0$ of $\left(\rho^{\mu}, \mathbf{m}^{\mu}\right)$ in the proof. Fix $\triangle t>0$. We divide the proof into two steps.

1. For $t \in[0, T-\triangle t]$, set

$$
w(t, \cdot):=\rho(t+\triangle t, \cdot)-\rho(t, \cdot) .
$$

Then, for any $\varphi(t, \mathbf{x}) \in C^{\infty}\left([0, T) \times \mathbb{T}_{P}\right)$ that is periodic in $\mathbf{x} \in \mathbb{R}^{3}$ with period $\mathbb{T}_{P}$, we have

$$
\begin{aligned}
\int_{\mathbb{T}_{P}} w(t, \mathbf{x}) \varphi(t, \mathbf{x}) \mathrm{d} \mathbf{x} & =\int_{t}^{t+\triangle t} \int_{\mathbb{T}_{P}} \partial_{s} \rho(s, \mathbf{x}) \varphi(t, \mathbf{x}) \mathrm{d} \mathbf{x} \mathrm{ds} \\
& =\int_{t}^{t+\triangle t} \int_{\mathbb{T}_{P}} \mathbf{m}(s, \mathbf{x}) \cdot \nabla \varphi(t, \mathbf{x}) \mathrm{d} \mathbf{x} \mathrm{ds} .
\end{aligned}
$$

By approximation, equality (4.2) still holds for

$$
\varphi \in L^{\infty}\left(0, T ; H^{1}\left(\mathbb{T}_{P}\right)\right) \cap C\left([0, T] ; L^{2}\left(\mathbb{T}_{P}\right)\right) \cap L^{\infty}\left([0, T) \times \mathbb{T}_{P}\right) .
$$

Choose

$$
\varphi=\varphi^{\delta}(t, \mathbf{x}):=\left(j_{\delta} * w\right)(t, \mathbf{x})=\int j_{\delta}(\mathbf{x}-\mathbf{y}) \operatorname{sign}(w(t, \mathbf{y}))|w(t, \mathbf{y})|^{\gamma-1} \mathrm{~d} \mathbf{y} \in \mathbb{R}^{3} \quad \text { for } \delta>0,
$$

which is periodic in $\mathbf{x} \in \mathbb{R}^{3}$ with period $\mathbb{T}_{P}$, where $j_{\delta}(\mathbf{x})=\frac{1}{\delta^{3}} j\left(\frac{\mathbf{x}}{\delta}\right) \geq 0$ is a standard mollifier with

$$
j \in C_{0}^{\infty}\left(\mathbb{R}^{3}\right), \quad \int j(\mathbf{x}) \mathrm{d} \mathbf{x}=1 .
$$


Then, for any $\mathbf{x} \in \mathbb{T}_{P}$, we have

$$
\begin{aligned}
\left\|\varphi^{\delta}\right\|_{L^{\infty}\left(\mathbb{T}_{P}\right)} & \leq \frac{1}{\delta^{3}} \int_{|\mathbf{x}-\mathbf{y}| \leq \delta} j\left(\frac{\mathbf{x}-\mathbf{y}}{\delta}\right)|w(t, \mathbf{y})|^{\gamma-1} \mathrm{~d} \mathbf{y} \\
& \leq \frac{M_{T}}{\delta^{3-\frac{3}{\gamma}}}\|j\|_{L^{\gamma}}\|w(t, \cdot)\|_{L^{\gamma}\left(\mathbb{T}_{P}\right)}^{\gamma-1} \leq \frac{M_{T}}{\delta^{3-\frac{3}{\gamma}}}
\end{aligned}
$$

Similarly, we have

$$
\begin{aligned}
\left\|\nabla \varphi^{\delta}\right\|_{L^{\infty}\left(\mathbb{T}_{P}\right)} & \leq \frac{1}{\delta^{4}} \int_{|\mathbf{x}-\mathbf{y}| \leq \delta}\left|j^{\prime}\left(\frac{\mathbf{x}-\mathbf{y}}{\delta}\right)\right||w(t, \mathbf{y})|^{\gamma-1} \mathrm{~d} \mathbf{y} \\
& \leq \frac{M}{\delta^{4-\frac{3}{\gamma}}}\left\|j^{\prime}\right\|_{L^{\gamma}}\|w(t, \cdot)\|_{L^{\gamma}\left(\mathbb{T}_{P}\right)}^{\gamma-1} \leq \frac{M}{\delta^{4-\frac{3}{\gamma}}} .
\end{aligned}
$$

As before, we use $M_{T}>0$ as a universal constant independent of $\mu>0$.

Integrating (4.2) in $t$ over $[0, T-\triangle t)$ with $\varphi=\varphi^{\delta}(t, \mathbf{x})$, we have

$$
\begin{aligned}
\int_{0}^{T-\triangle t} \int_{\mathbb{T}_{P}}|w(t, \mathbf{x})|^{\gamma} \mathrm{d} \mathbf{x} \mathrm{dt}= & \int_{0}^{T-\triangle t} \int_{t}^{t+\triangle t} \int_{\mathbb{T}_{P}} \mathbf{m}(s, \mathbf{x}) \cdot \nabla \varphi^{\delta}(t, \mathbf{x}) \mathrm{d} \mathbf{x} \mathrm{d} s \mathrm{dt} \\
& +\int_{0}^{T-\triangle t} \int_{\mathbb{T}_{P}}\left(|w(t, \mathbf{x})|^{\gamma}-w(t, \mathbf{x}) \varphi^{\delta}(t, \mathbf{x})\right) \mathrm{d} \mathbf{x} \mathrm{dt} \\
= & : I_{1}^{\delta}+I_{2}^{\delta} .
\end{aligned}
$$

Then

$$
\left|I_{1}^{\delta}\right| \leq \frac{M_{T} \triangle t}{\delta^{4-\frac{3}{\gamma}}}\|\mathbf{m}(t, \cdot)\|_{L^{1}\left(\mathbb{T}_{P}\right)} \leq \frac{M_{T} \triangle t}{\delta^{4-\frac{3}{\gamma}}}\|\rho\|_{L^{1}\left(\mathbb{T}_{P}\right)}^{\frac{1}{2}}\|\sqrt{\rho} \mathbf{u}\|_{L^{2}\left(\mathbb{T}_{P}\right)} \leq \frac{M_{T} \Delta t}{\delta^{4-\frac{3}{\gamma}}} .
$$

Furthermore, for $I_{2}^{\delta}$, we have

$$
\begin{aligned}
& \left|I_{2}^{\delta}\right|=\left|\int_{0}^{T-\Delta t} \int_{\mathbb{T}_{P}}\left(\int_{\mathbb{R}^{3}} j_{\delta}(\mathbf{x}-\mathbf{y})\left(|w(t, \mathbf{x})|^{\gamma}-w(t, \mathbf{x}) \operatorname{sign}(w(t, \mathbf{y}))|w(t, \mathbf{y})|^{\gamma-1}\right) \mathrm{d} \mathbf{y}\right) \mathrm{d} \mathbf{x} \mathrm{dt}\right| \\
& =\left|\int_{|\mathbf{y}| \leq 1} j(\mathbf{y})\left(\int_{0}^{T-\triangle t} \int_{\mathbb{T}_{P}}\left(|w(t, \mathbf{x})|^{\gamma}-w(t, \mathbf{x}) \operatorname{sign}(w(t, \mathbf{x}-\delta \mathbf{y}))|w(t, \mathbf{x}-\delta \mathbf{y})|^{\gamma-1}\right) \mathrm{d} \mathbf{x} \mathrm{d} t\right) \mathrm{d} \mathbf{y}\right| \\
& \leq \int_{|\mathbf{y}| \leq 1} j(\mathbf{y})\left(\left.\int_{0}^{T-\Delta t} \int_{\mathbb{T}_{P}}|| w(t, \mathbf{x})\right|^{\gamma}-|w(t, \mathbf{x}-\delta \mathbf{y})|^{\gamma} \mid \mathrm{d} \mathbf{x} \mathrm{dt}\right) \mathrm{d} \mathbf{y} \\
& +\left|\int_{|\mathbf{y}| \leq 1} j(\mathbf{y})\left(\int_{0}^{T-\Delta t} \int_{\mathbb{T}_{P}}(w(t, \mathbf{x})-w(t, \mathbf{x}-\delta \mathbf{y})) \operatorname{sign}(w(y, \mathbf{x}-\delta \mathbf{y}))|w(t, \mathbf{x}-\delta \mathbf{y})|^{\gamma-1} \mathrm{~d} \mathbf{x} \mathrm{dt}\right) \mathrm{d} \mathbf{y}\right| \\
& \leq \gamma \int_{|\mathbf{y}| \leq 1} j(\mathbf{y})\left(\int_{0}^{T-\Delta t} \int_{\mathbb{T}_{P}}|w(t, \mathbf{x})-w(t, \mathbf{x}-\delta \mathbf{y})|\left(|w(t, \mathbf{x})|^{\gamma-1}+|w(t, \mathbf{x}-\delta \mathbf{y})|^{\gamma-1}\right) \mathrm{d} \mathbf{x} \mathrm{dt}\right) \mathrm{d} \mathbf{y} \\
& +\int_{|\mathbf{y}| \leq 1} j(\mathbf{y})\left(\int_{0}^{T-\Delta t} \int_{\mathbb{T}_{P}}|w(t, \mathbf{x})-w(t, \mathbf{x}-\delta \mathbf{y})||w(t, \mathbf{x}-\delta \mathbf{y})|^{\gamma-1} \mathrm{~d} \mathbf{x} \mathrm{dt}\right) \mathrm{d} \mathbf{y} \\
& \leq M_{T}\|w\|_{L^{\gamma}\left([0, T] \times \mathbb{T}_{P}\right)}^{\gamma-1} \int_{|\mathbf{y}| \leq 1} j(\mathbf{y})\left(\int_{0}^{T-\triangle t} \int_{\mathbb{T}_{P}}|w(t, \mathbf{x})-w(t, \mathbf{x}-\delta \mathbf{y})|^{\gamma} \mathrm{d} \mathbf{x} \mathrm{dt}\right)^{\frac{1}{\gamma}} \mathrm{d} \mathbf{y} \\
& \leq M_{T} \delta^{\frac{\alpha_{1}}{\gamma}} \int_{|\mathbf{y}| \leq 1} j(\mathbf{y})|\mathbf{y}|^{\frac{\alpha_{1}}{\gamma}} \leq M_{T} \delta^{\frac{\alpha_{1}}{\gamma}}
\end{aligned}
$$

where we have used the fact that $\|w\|_{L^{\gamma}\left([0, T] \times \mathbb{T}_{P}\right)} \leq M_{T}$ from Proposition 3.2 , 
Combining (4.2)-(4.9), we have

$$
\int_{0}^{T-\triangle t} \int_{\mathbb{T}_{P}}|\mathbf{w}(t, \mathbf{x})|^{\gamma} \mathrm{d} \mathbf{x d t} \leq \mathrm{M} \inf _{\delta>0}\left\{\frac{\triangle \mathrm{t}}{\delta^{4-\frac{3}{\gamma}}}+\delta^{\frac{\alpha_{1}}{\gamma}}\right\} .
$$

Choose

$$
\delta=|\triangle t|^{\frac{\gamma}{4 \gamma+\alpha_{1}-3}} .
$$

We conclude

$$
\int_{0}^{T-\triangle t} \int_{\mathbb{T}_{P}}|\mathbf{w}(t, \mathbf{x})|^{\gamma} \mathrm{d} \mathbf{x d t} \leq \mathrm{M}(\triangle \mathrm{t})^{\frac{\alpha_{1}}{4 \gamma-3+\alpha_{1}}}
$$

2. Now we estimate $\mathbf{m}(t, \mathbf{x})$. For $t \in[0, T-\triangle t]$, set

$$
\mathbf{w}(t, \cdot)=\mathbf{m}(t+\triangle t, \cdot)-\mathbf{m}(t, \cdot) .
$$

Then, for any $\varphi(t, \mathbf{x}) \in C^{\infty}\left([0, T) \times \mathbb{T}_{P}\right)$ that is periodic in $\mathbf{x} \in \mathbb{R}^{3}$ with period $\mathbb{T}_{P}$, we have

$$
\begin{aligned}
\int_{\mathbb{T}_{P}} \mathbf{w}(t, \mathbf{x}) \cdot \boldsymbol{\varphi}(t, \mathbf{x}) \mathrm{d} \mathbf{x}= & \int_{t}^{t+\Delta t} \int_{\mathbb{T}_{P}} \partial_{s} \mathbf{m}(s, \mathbf{x}) \cdot \boldsymbol{\varphi}(t, \mathbf{x}) \mathrm{d} \mathbf{x} \mathrm{d} \mathbf{s} \\
= & \int_{t}^{t+\triangle t} \int_{\mathbb{T}_{P}}(\rho \mathbf{u} \otimes \mathbf{u})(s, \mathbf{x}): \nabla \boldsymbol{\varphi}(t, \mathbf{x}) \mathrm{d} \mathbf{x} \mathrm{d} \mathbf{s} \\
& -\int_{t}^{t+\triangle t} \int_{\mathbb{T}_{P}} \Sigma(s, \mathbf{x}): \nabla \boldsymbol{\varphi}(t, \mathbf{x}) \mathrm{d} \mathbf{x} \mathrm{d} s \\
& +\int_{t}^{t+\triangle t} \int_{\mathbb{T}_{P}} p(s, \mathbf{x}) \nabla \cdot \boldsymbol{\varphi}(t, \mathbf{x}) \mathrm{d} \mathbf{x} \mathrm{d} s \\
& +\int_{t}^{t+\Delta t} \int_{\mathbb{T}_{P}}(\rho \mathbf{f})(s, \mathbf{x}) \cdot \varphi(t, \mathbf{x}) \mathrm{d} \mathbf{x} \mathrm{ds},
\end{aligned}
$$

where $\nabla \boldsymbol{\varphi}=\left(\partial_{x_{j}} \varphi_{i}\right)$ is the $3 \times 3$ matrix.

By approximation, equality (4.11) still holds for

$$
\boldsymbol{\varphi} \in L^{\infty}\left(0, T ; H^{1}\left(\mathbb{T}_{P}\right)\right) \cap C\left([0, T] ; L^{2}\left(\mathbb{T}_{P}\right)\right) \cap L^{\infty}\left([0, T) \times \mathbb{T}_{P}\right) .
$$

Choose

$$
\boldsymbol{\varphi}=\varphi^{\delta}(t, \mathbf{x}):=\left(j_{\delta} * \mathbf{w}\right)(t, \mathbf{x})=\int j_{\delta}(\mathbf{y}) \mathbf{w}(t, \mathbf{x}-\mathbf{y}) \mathrm{d} \mathbf{y} \in \mathbb{R}^{3} \quad \text { for } \delta>0,
$$

which is periodic in $\mathbf{x} \in \mathbb{R}^{3}$ with period $\mathbb{T}_{P}$, where $j_{\delta}(\mathbf{x})=\frac{1}{\delta^{3}} j\left(\frac{\mathbf{x}}{\delta}\right) \geq 0$ is the standard mollifier with (4.4).

Then, for any $\mathbf{x} \in \mathbb{T}_{P}$, we have

$$
\begin{aligned}
\left\|\boldsymbol{\varphi}_{\delta}\right\|_{L^{\infty}\left(\mathbb{T}_{P}\right)} & \leq \frac{1}{\delta^{3}} \int_{|\mathbf{x}-\mathbf{y}| \leq \delta} j\left(\frac{\mathbf{x}-\mathbf{y}}{\delta}\right)|\mathbf{w}(t, \mathbf{y})| \mathrm{d} \mathbf{y} \leq \frac{\mathrm{M}_{\mathrm{T}}}{\delta^{3}}\left\|_{\mathrm{j}}\right\|_{\mathrm{L}^{\infty}}\|\sqrt{\rho(\mathrm{t}, \cdot)}(\sqrt{\rho} \mathbf{u})(\mathrm{t}, \cdot)\|_{\mathrm{L}^{1}\left(\mathbb{T}_{\mathrm{P}}\right)} \\
& \leq \frac{M_{T}}{\delta^{3}}\|j\|_{L^{\infty}}\|\rho(t, \cdot)\|_{L^{1}}\|(\sqrt{\rho} \mathbf{u})(t, \cdot)\|_{L^{2}\left(\mathbb{T}_{P}\right)} \leq \frac{M_{T}}{\delta^{3}}
\end{aligned}
$$

Similarly, we have

$$
\left\|\nabla \varphi_{\delta}\right\|_{L^{\infty}\left(\mathbb{T}_{P}\right)} \leq \frac{1}{\delta^{4}} \int_{|\mathbf{x}-\mathbf{y}| \leq \delta}\left|j^{\prime}\left(\frac{\mathbf{x}-\mathbf{y}}{\delta}\right)\right||\mathbf{w}(t, \mathbf{y})| \mathrm{d} \mathbf{y} \leq \frac{\mathrm{M}_{\mathrm{T}}}{\delta^{4}}\left\|\mathrm{j}^{\prime}\right\|_{\mathrm{L}^{\infty}}\|\mathbf{w}(\mathrm{t}, \cdot)\|_{\mathrm{L}^{1}\left(\mathbb{T}_{\mathrm{P}}\right)} \leq \frac{\mathrm{M}_{\mathrm{T}}}{\delta^{4}} .
$$


Integrating (4.11) in $t$ over $[0, T-\triangle t)$ with $\varphi=\varphi^{\delta}(t, \mathbf{x})$ and using (4.13), we have

$$
\begin{aligned}
& \int_{0}^{T-\triangle t} \int_{\mathbb{T}_{P}}|\mathbf{w}(t, \mathbf{x})|^{2} \mathrm{~d} \mathbf{x} \mathrm{dt} \\
& =\int_{0}^{T-\Delta t} \int_{\mathbb{T}_{P}} \mathbf{w}(t, \mathbf{x}) \cdot\left(\mathbf{w}(t, \mathbf{x})-\varphi^{\delta}(t, \mathbf{x})\right) \mathrm{d} \mathbf{x} \mathrm{dt} \\
& +\int_{0}^{T-\Delta t} \int_{t}^{t+\Delta t} \int_{\mathbb{T}_{P}}(\rho \mathbf{u} \otimes \mathbf{u})(s, \mathbf{x}): \nabla \varphi^{\delta}(t, \mathbf{x}) \mathrm{d} \mathbf{x} \mathrm{d} s \mathrm{dt} \\
& -\int_{0}^{T-\Delta t} \int_{\mathbb{T}_{P}} \int_{t}^{t+\Delta t} \Sigma(s, \mathbf{x}): \nabla \varphi^{\delta}(t, \mathbf{x}) \mathrm{dsd} \mathbf{x d t} \\
& +\int_{0}^{T-\Delta t} \int_{\mathbb{T}_{P}} \int_{t}^{t+\Delta t} p(s, \mathbf{x}) \nabla \cdot \varphi^{\delta}(t, \mathbf{x}) \mathrm{dsd} \mathbf{x} \mathrm{dt} \\
& +\int_{0}^{T-\Delta t} \int_{t}^{t+\Delta t} \int_{\mathbb{T}_{P}}(\rho \mathbf{f})(s, \mathbf{x}) \cdot \varphi^{\delta}(t, \mathbf{x}) \mathrm{d} \mathbf{x d s d t} \\
& =: J_{1}^{\delta}+J_{2}^{\delta}+J_{3}^{\delta}+J_{4}^{\delta}+J_{5}^{\delta} \text {. }
\end{aligned}
$$

For $J_{1}^{\delta}$, we have

$$
\begin{aligned}
\left|J_{1}^{\delta}\right|= & \left|\int_{0}^{T-\triangle t} \int_{\mathbb{T}_{P}}\left(\int_{\mathbb{R}^{3}} j_{\delta}(\mathbf{x}-\mathbf{y})\left(|\mathbf{w}(t, \mathbf{x})|^{2}-\mathbf{w}(t, \mathbf{x}) \mathbf{w}(t, \mathbf{y})\right) \mathrm{d} \mathbf{y}\right) \mathrm{d} \mathbf{x} \mathrm{dt}\right| \\
= & \int_{|\mathbf{y}| \leq 1} j(\mathbf{y})\left(\int_{0}^{T-\triangle t} \int_{\mathbb{T}_{P}}\left(|\mathbf{w}(t, \mathbf{x})|^{2}-\mathbf{w}(t, \mathbf{x}) \mathbf{w}(t, \mathbf{x}-\delta \mathbf{y})\right) \mathrm{d} \mathbf{x} \mathrm{dt}\right) \mathrm{d} \mathbf{y} \\
\leq & \int_{|\mathbf{y}| \leq 1} j(\mathbf{y})\left(\int_{0}^{T-\triangle t} \int_{\mathbb{T}_{P}}|\mathbf{w}(t, \mathbf{x})-\mathbf{w}(t, \mathbf{x}-\delta \mathbf{y})|(|\mathbf{w}(t, \mathbf{x})|+|\mathbf{w}(t, \mathbf{x}-\delta \mathbf{y})|) \mathrm{d} \mathbf{x d t}\right) \mathrm{d} \mathbf{y} \\
& +\int_{|\mathbf{y}| \leq 1} j(\mathbf{y})\left(\int_{0}^{T-\triangle t} \int_{\mathbb{T}_{P}}|\mathbf{w}(t, \mathbf{x})-\mathbf{w}(t, \mathbf{x}-\delta \mathbf{y}) \| \mathbf{w}(t, \mathbf{x}-\delta \mathbf{y})| \mathrm{d} \mathbf{x} \mathrm{dt}\right) \mathrm{d} \mathbf{y} \\
\leq & M_{T}\|\mathbf{w}\|_{L^{2}\left([0, T] \times \mathbb{T}_{P}\right)} \int_{|\mathbf{y}| \leq 1} j(\mathbf{y})\left(\int_{0}^{T-\triangle t} \int_{\mathbb{T}_{P}}|\mathbf{w}(t, \mathbf{x})-\mathbf{w}(t, \mathbf{x}-\delta \mathbf{y})|^{2} \mathrm{~d} \mathbf{x d t}\right)^{\frac{1}{2}} \mathrm{~d} \mathbf{y} \\
\leq & M_{T} \delta^{\frac{\alpha_{1}}{2}} \int_{|\mathbf{y}| \leq 1} j(\mathbf{y})|\mathbf{y}|^{\frac{\alpha}{2}} d \mathbf{y} \leq M_{T} \delta^{\frac{\alpha_{1}}{2}},
\end{aligned}
$$

where we have used the fact that $\|\mathbf{w}\|_{L^{2}\left([0, T] \times \mathbb{T}_{P}\right)} \leq M_{T}$ from Proposition 3.2 .

For $J_{2}^{\delta}$ and $J_{3}^{\delta}$, we find

$$
\left|J_{2}^{\delta}\right| \leq \frac{M_{T} \Delta t}{\delta^{4}}\|\sqrt{\rho} \mathbf{u}\|_{L^{1}\left(\mathbb{T}_{P}\right)}^{2} \leq \frac{M_{T} \Delta t}{\delta^{4}} \mathcal{E}_{0} \leq \frac{M_{T} \Delta t}{\delta^{4}}
$$


and

$$
\begin{aligned}
\left|J_{3}^{\delta}\right| & \leq \frac{M_{T}}{\delta^{4}} \int_{0}^{T-\Delta t} \int_{\mathbb{T}_{P}} \int_{t}^{t+\Delta t}|\Sigma| \mathrm{d} \mathbf{x d s d t} \\
& \leq \frac{M_{T} \Delta t}{\delta^{4}} \int_{0}^{T-\Delta t} \int_{\mathbb{T}_{P}}|\Sigma| \mathrm{d} \mathbf{x d s} \\
& \leq \frac{M_{T} \Delta t}{\delta^{4}}\left(\int_{0}^{T-\Delta t} \int_{\mathbb{T}_{P}}|\Sigma|^{2} \mathrm{~d} \mathbf{x d s}\right)^{2} \\
& \leq \frac{M_{T} \Delta t}{\delta^{4}}
\end{aligned}
$$

since $\max \{\mu,|\lambda|\} \leq \mu_{0}$ for some fixed $\mu_{0}$ throughout the paper.

For $J_{4}^{\delta}$ and $J_{5}^{\delta}$, we see

$$
\left|J_{4}^{\delta}\right| \leq \frac{M_{T} \Delta t}{\delta^{4}}\|\rho\|_{L^{\gamma}\left([0, T] \times \mathbb{T}_{P}\right)}^{\gamma} \leq \frac{M_{T} \Delta t}{\delta^{4}}
$$

and

$$
\left|J_{5}^{\delta}\right| \leq M_{T} \Delta t\|\rho \mathbf{f}\|_{L^{1}}\left\|\varphi^{\delta}\right\|_{L^{\infty}} \leq \frac{M_{T} \Delta t}{\delta^{3}}\|\rho\|_{L^{\gamma}\left(\mathbb{T}_{P}\right)}\|\mathbf{f}\|_{L^{1}\left(0, T ; L^{\frac{2 \gamma}{\gamma-1}}\left(\mathbb{T}_{P}\right)\right)} \leq \frac{M_{T} \Delta t}{\delta^{3}} .
$$

Therefore, combining (4.14)-(4.19) together, we have

$$
\int_{0}^{T-\Delta t} \int_{\mathbb{T}_{P}}|\mathbf{w}(t, \mathbf{x})|^{2} \mathrm{~d} \mathbf{x} \mathrm{dt} \leq \mathrm{M}_{\mathrm{T}}\left(\delta^{\frac{\alpha_{1}}{2}}+\frac{\Delta \mathrm{t}}{\delta^{4}}+\frac{\Delta \mathrm{t}}{\delta^{3}}\right) \leq \mathrm{M}_{\mathrm{T}}\left(\delta^{\frac{\alpha_{1}}{2}}+\frac{\Delta \mathrm{t}}{\delta^{4}}\right) \leq \mathrm{M}_{\mathrm{T}}(\Delta \mathrm{t})^{\frac{\alpha_{1}}{8+\alpha_{1}}} .
$$

Choosing

we complete the proof.

$$
\alpha_{2}=\min \left\{\frac{\alpha_{1}}{4 \gamma-3+\alpha_{1}}, \frac{\alpha_{1}}{8+\alpha_{1}}\right\}
$$

As a direct corollary, we have

Proposition 4.2. Under Assumption (CKHw), for any $T \in(0, \infty)$, there exists $M_{T}>0$, independent of $\mu>0$, such that

$$
\int_{0}^{T} \int_{\mathbb{T}_{P}}\left(\left|\left(D_{t}^{\frac{\alpha_{2}}{\gamma}}, D_{\mathbf{x}}^{\frac{\alpha_{1}}{\gamma}}\right) \rho^{\mu}(t, \mathbf{x})\right|^{\gamma}+\left|\left(D_{t}^{\frac{\alpha_{2}}{2}}, D_{\mathbf{x}}^{\frac{\alpha_{1}}{2}}\right) \mathbf{m}^{\mu}(t, \mathbf{x})\right|^{2}\right) \mathrm{d} \mathbf{x d t} \leq \mathrm{M}_{\mathrm{T}}<\infty
$$

for some $\alpha_{1}>0$ and $\alpha_{2}=\min \left\{\frac{\alpha_{1}}{4 \gamma-3+\alpha_{1}}, \frac{\alpha_{1}}{8+\alpha_{1}}\right\}>0$.

Combining Proposition 3.1 with Proposition 4.2, we have

$$
\left\|\rho^{\mu}\right\|_{W^{\hat{\alpha}_{1}, \gamma\left([0, T) \times \mathbb{T}_{P}\right)}}+\left\|\mathbf{m}^{\mu}\right\|_{H^{\hat{\alpha}_{2}\left([0, T) \times \mathbb{T}_{P}\right)}} \leq M_{T}<\infty,
$$

where $\hat{\alpha}_{1}=\frac{1}{\gamma} \min \left\{\alpha_{1}, \alpha_{2}\right\}$ and $\hat{\alpha}_{2}=\frac{1}{2} \min \left\{\alpha_{1}, \alpha_{2}\right\}$. Then, by the Sobolev imbedding theorem, we have

Proposition 4.3. Under Assumption (CKHw), for any $T \in(0, \infty)$, there exists $M_{T}>0$, independent of $\mu>0$, such that

$$
\left\|\rho^{\mu}\right\|_{L^{q_{1}\left([0, T) \times \mathbb{T}_{P}\right)}}+\left\|\mathbf{m}^{\mu}\right\|_{L^{q_{2}\left([0, T) \times \mathbb{T}_{P}\right)}} \leq M_{T}<\infty
$$

for some $q_{1}>\gamma$ and $q_{2}>2$. 


\section{INVISCID LIMIT}

In this section, we show the inviscid limit from the compressible Navier-Stokes to the Euler equations under Assumption (CKHw).

Theorem 5.1. The compressible Kolmogorov-type hypothesis, Assumption (CKHw), implies the strong compactness in $\left(L^{q_{1}} \times L^{q_{2}}\right)\left([0, T) \times \mathbb{T}_{P}\right)$ of the solutions $\left(\rho^{\mu}, \mathbf{m}^{\mu}\right)(t, \mathbf{x})$ of the compressible Navier-Stoke equations (1.1) in $\mathbb{R}_{T}^{3}$ in the sense of Definition 1.1 when the viscosity $\mu$ tends to zero, for some $q_{1}>\gamma$ and $q_{2}>2$. That is, there exist a subsequence (still denoted) $\left(\rho^{\mu}, \mathbf{m}^{\mu}\right)(t, \mathbf{x})$ and a function $(\rho, \mathbf{m}) \in\left(L^{q_{1}} \times L^{q_{2}}\right)\left([0, T) \times \mathbb{T}_{P}\right)$ such that

$$
\left(\rho^{\mu}, \mathbf{m}^{\mu}\right)(t, \mathbf{x}) \rightarrow(\rho, \mathbf{m})(t, \mathbf{x}) \quad \text { a.e. as } \mu \rightarrow 0,
$$

and $(\rho, \mathbf{m})(t, \mathbf{x})$ is a weak solution of the compressible Euler equations (1.14) with Cauchy data $\left(\rho_{0}, \mathbf{m}_{0}\right)(\mathbf{x})$ in the sense of Definition 1.2 .

Proof. We divide the proof into six steps.

1. Propositions 3.2 and 4.1 imply the $\left(L^{\gamma} \times L^{2}\right)$-equicontinuity of $\left(\rho^{\mu}, \mathbf{m}^{\mu}\right)(t, \mathbf{x})$ in $(t, \mathbf{x}) \in$ $[0, T) \times \mathbb{T}_{P}$, independent of $\mu>0$. This yields that there exist both a subsequence (still denoted $)\left(\rho^{\mu}, \mathbf{m}^{\mu}\right)(t, \mathbf{x})$ and a function $(\rho, \mathbf{m})(t, \mathbf{x}) \in L^{\gamma} \times L^{2}$ such that

$$
\left(\rho^{\mu}, \mathbf{m}^{\mu}\right)(t, \mathbf{x}) \rightarrow(\rho, \mathbf{m})(t, \mathbf{x}) \quad \text { in } L^{\gamma} \times L^{2} \text { as } \mu \rightarrow 0,
$$

which implies that

$$
\left(\rho^{\mu}, \mathbf{m}^{\mu}\right)(t, \mathbf{x}) \rightarrow(\rho, \mathbf{m})(t, \mathbf{x}) \quad \text { a.e. as } \mu \rightarrow 0 \text {. }
$$

2. From Proposition 4.3, we have

$$
(\rho, \mathbf{m}) \in\left(L^{q_{1}} \times L^{q_{2}}\right)\left([0, T) \times \mathbb{T}_{P}\right)
$$

and

$$
\left(\rho^{\mu}, \mathbf{m}^{\mu}\right)(t, \mathbf{x}) \rightarrow(\rho, \mathbf{m})(t, \mathbf{x}) \quad \text { in }\left(L^{p_{1}} \times L^{p_{2}}\right)\left([0, T) \times \mathbb{T}_{P}\right) \text { as } \mu \rightarrow 0 .
$$

for any $p_{1} \in\left[1, q_{1}\right)$ and $p_{2} \in\left[1, q_{2}\right)$.

3. Proposition 3.2 implies that

$$
\mathbf{M}^{\mu}:=\rho^{\mu} \mathbf{u}^{\mu} \otimes \mathbf{u}^{\mu} \rightarrow \mathbf{M} \in L^{q} \quad \text { weakly in } L^{q} \text { for some } q>1 .
$$

Since

$$
\mathbf{m}^{\mu} \otimes \mathbf{m}^{\mu}=\mathbf{M}^{\mu} \rho^{\mu}
$$

then the limits satisfy

$$
\mathbf{m} \otimes \mathbf{m}=\mathbf{M} \rho \quad \text { a.e. }
$$

which implies that $\mathbf{m} \otimes \mathbf{m}$ is absolutely continuous with respect to $\rho$ with an $L^{q}$ density, uniformly in $\rho(t, \mathbf{x}) \geq 0$. That is, $\frac{\mathbf{m} \otimes \mathbf{m}}{\rho}$ is well-defined even on the vacuum $\{\rho(t, \mathbf{x})=0\}$, and $\frac{\mathbf{m} \otimes \mathbf{m}}{\rho}=\mathbf{M}$ a.e. on the non-vacuum state $\{\rho(t, \mathbf{x})>0\}$. Therefore, we define

$$
\frac{\mathbf{m} \otimes \mathbf{m}}{\rho}=\mathbf{M} \in L^{q} \quad \text { for } q>1 .
$$

This implies that

$$
\frac{\mathbf{m}^{\mu} \otimes \mathbf{m}^{\mu}}{\rho^{\mu}}(t, \mathbf{x}) \rightarrow \frac{\mathbf{m} \otimes \mathbf{m}}{\rho}(t, \mathbf{x}) \in L^{q} \quad \text { a.e. as } \mu \rightarrow 0
$$


for $q>1$.

4. Similarly, we notice that

$$
V^{\mu}:=\rho^{\mu}\left|\mathbf{u}^{\mu}\right|^{2} \rightarrow V \in L^{q} \quad \text { weakly in } L^{q}, q>1 .
$$

Since

$$
\left|\mathbf{m}^{\mu}\right|^{2}=V^{\mu} \rho^{\mu},
$$

then the limits satisfy

$$
|\mathbf{m}|^{2}=V \rho \quad \text { a.e. }
$$

which implies that $|\mathbf{m}|^{2}$ is absolutely continuous with respect to $\rho$ with an $L^{q}$ density, uniformly in $\rho(t, \mathbf{x}) \geq 0$. That is, $\frac{|\mathbf{m}|^{2}}{\rho}$ is well-defined even on the vacuum $\{\rho(t, \mathbf{x})=0\}$, and $\frac{|\mathbf{m}|^{2}}{\rho}=V$ a.e. on the non-vacuum state $\{\rho(t, \mathbf{x})>0\}$. Therefore, we define

$$
\frac{|\mathbf{m}|^{2}}{\rho}=V \in L^{q} \quad \text { for } q>1 \text {. }
$$

This implies that

$$
\frac{\left|\mathbf{m}^{\mu}\right|^{2}}{\rho^{\mu}}(t, \mathbf{x}) \rightarrow \frac{|\mathbf{m}|^{2}}{\rho}(t, \mathbf{x}) \in L^{q} \quad \text { a.e. as } \mu \rightarrow 0
$$

for $q>1$.

5. For any $\varphi \in C_{0}^{\infty}\left(\mathbb{R}_{T}^{3} ; \mathbb{R}\right)$, we multiply both sides $(1.1)$ by $\varphi$ and integrate over $\mathbb{R}_{T}^{3}$ to obtain

$$
\int_{0}^{T} \int_{\mathbb{R}^{3}}\left(\rho^{\mu} \varphi_{t}+\mathbf{m}^{\mu} \cdot \nabla \varphi\right) \mathrm{d} \mathbf{x d t}+\int_{\mathbb{R}^{3}} \rho_{0}(\mathbf{x}) \varphi(0, \mathbf{x}) \mathrm{d} \mathbf{x}=0 .
$$

Letting $\mu \rightarrow 0$, we conclude (1.15).

6. For any $\varphi \in C_{0}^{\infty}\left(\mathbb{R}_{T}^{3} ; \mathbb{R}^{3}\right.$ ), we multiply both sides $(1.1)$ by $\varphi$ and integrate over $\mathbb{R}_{T}^{3}$ to obtain

$$
\begin{aligned}
& \int_{0}^{T} \int_{\mathbb{R}^{3}}\left(\mathbf{m}^{\mu} \cdot \boldsymbol{\varphi}_{t}+\frac{\mathbf{m}^{\mu} \otimes \mathbf{m}^{\mu}}{\rho^{\mu}}: \nabla \boldsymbol{\varphi}+p\left(\rho^{\mu}\right) \operatorname{div} \boldsymbol{\varphi}+\rho^{\mu} \mathbf{f} \cdot \boldsymbol{\varphi}\right) \mathrm{d} \mathbf{x d t}+\int_{\mathbb{R}^{3}} \mathbf{m}_{0}(\mathbf{x}) \cdot \boldsymbol{\varphi}(0, \mathbf{x}) \mathrm{d} \mathbf{x} \\
& =-\int_{0}^{T} \int_{\mathbb{R}^{3}} \Sigma^{\mu}: \nabla \boldsymbol{\varphi} \mathrm{d} \mathbf{x d t} \\
& =-2 \mu \int_{0}^{T} \int_{\mathbb{R}^{3}} D\left(\nabla \mathbf{u}^{\mu}\right): \nabla \boldsymbol{\varphi} \mathrm{d} \mathbf{x} \mathrm{dt}-\lambda \int_{0}^{\mathrm{T}} \int_{\mathbb{R}^{3}}\left(\nabla \cdot \mathbf{u}^{\mu}\right)^{2} \operatorname{Tr}(\nabla \boldsymbol{\varphi}) \mathrm{d} \mathbf{x} \mathrm{dt} .
\end{aligned}
$$

Notice that

$$
\begin{aligned}
& \left|\mu \int_{0}^{T} \int_{\mathbb{R}^{3}} D\left(\nabla \mathbf{u}^{\mu}\right): \nabla \boldsymbol{\varphi} \mathrm{d} \mathbf{x d t}\right| \leq \mathrm{M}_{\mathrm{T}} \sqrt{\mu}\left\|\sqrt{\mu} \nabla \mathbf{u}^{\mu}\right\|_{\mathrm{L}^{2}}\|\nabla \boldsymbol{\varphi}\|_{\mathrm{L}^{2}}^{2} \leq \sqrt{\mu} \mathrm{M}_{\mathrm{T}} \\
& \left|\lambda \int_{0}^{T} \int_{\mathbb{R}^{3}}\left(\nabla \cdot \mathbf{u}^{\mu}\right)^{2} \operatorname{Tr}(\Delta \boldsymbol{\varphi}) \mathrm{d} \mathbf{x d t}\right| \leq \mathrm{M}_{\mathrm{T}} \sqrt{|\lambda|}\left\|\sqrt{|\lambda|} \nabla \cdot \mathbf{u}^{\mu}\right\|_{\mathrm{L}^{2}}\|\nabla \varphi\|_{\mathrm{L}^{2}}^{2} \leq \sqrt{|\lambda|} \mathrm{M}_{\mathrm{T}} .
\end{aligned}
$$

Letting $\mu \rightarrow 0$, we conclude (1.16). 
Integrating (1.11) over $[0, T) \times \mathbb{T}_{P}$ yields

$$
\begin{aligned}
\int_{\mathbb{T}_{P}} E^{\mu}(t, \mathbf{x}) \mathrm{d} \mathbf{x} & =\int_{\mathbb{T}_{P}}\left(\frac{1}{2} \frac{\left|\mathbf{m}^{\mu}\right|^{2}}{\rho}+\frac{\rho^{\mu} c\left(\rho^{\mu}\right)}{\gamma-1}\right)(t, \mathbf{x}) \mathrm{d} \mathbf{x} \\
& \leq \int_{\mathbb{T}_{P}} E_{0}(\mathbf{x}) \mathrm{d} \mathbf{x}+\int_{0}^{\mathrm{t}} \int_{\mathbb{T}_{P}} \mathbf{m}^{\mu}(\mathrm{s}, \mathbf{x}) \cdot \mathbf{f}(\mathrm{s}, \mathbf{x}) \mathrm{d} \mathbf{x} \mathrm{ds} .
\end{aligned}
$$

Letting $\mu \rightarrow 0$, we conclude (1.17).

Combining (1.15) (1.17) together yields that $(\rho, \mathbf{m})(t, \mathbf{x})$ is a weak solution of the Euler equations (1.14) with Cauchy data (1.2) in the sense of Definition 1.2. This completes the proof.

\section{Comments on the Main Results from a Physics Perspective}

While the extension of these results to general $\beta>0$ is interesting, the deepest conclusions

result in the extensions beyond the K41 exponent $-\frac{5}{3}$. For incompressible turbulence, such $\beta$ values lead to a fractal dimension $d_{f}<3$ of turbulence. In other words, turbulence occurs on a fractal set of dimension strictly less than 3. In fact, there are two fractal sets for turbulence, associated with the strain rate and the enthalpy ( $c f$. [22]), with values $d_{f}=2.61 \pm 0.03$ for the strain rate fractal and $d_{f}=2.22 \pm 0.03$ for the enstrophy fractal. These estimates result from the analysis of the finely resolved turbulence data base ( $c f . \quad 32$, 44]). The value for the strain rate fractal is consistent with a variety of simulation and experimental values reported by others (e.g. [19]), while the enstrophy fractal has received less prior attention ( $c f$. [2]). Multifractal theories ( $c f$. [19]) for incompressible turbulence identify a continuous infinity of additional fractal sets derived from the basic ones of strictly decreasing fractal dimensions and associated with the higher order statistics of the two point correlation function. Multifractal ideas motivate a number of simulation studies of compressible turbulence, as discussed in the introduction.

It is a strict mathematical result for the incompressible Euler equations with (CKHi) (i.e., $\beta>\frac{2}{3}$, corresponding to the Kolmogorov exponent $\frac{5}{3}$ ) that the total system energy is constant as a function of time ( $c f$. [4]).

An extension of the Kolmogorov hypothesis (K62) in [27] and its corrections to compressible turbulence is a plausible conjecture, and adds weight to our belief in the existence and uniqueness of the compressible Euler equations in a regime of fully developed turbulence. We do not assert that these hypotheses apply to all solutions of the compressible Euler equations. The Taylor-Green vortex, which does not possess a K41 cascade of turbulent scales, but rather consists of a single large vortex, is postulated [37] to be a fundamental singularity for the Euler equations in time $t<\infty$. Reasoning against this singularity is found in [20]. Recent high resolution simulation on the solution of the Taylor-Green vortex problem can be found in [10] (also see [2]).

The simulation does not show a singularity, but rather an eventual transition from the isolated vortex to a generalized turbulent flow, as predicted in [20]. The strictly axisymmetric vortex is unstable and, as the instability is approached, it is increasingly unstable to nonaxisymmetric perturbations. As an isolated unstable point singularity in the space of initial conditions, it is "removable", and the solution, starting exactly at this isolated point of initial conditions, can be extended (nonuniquely) beyond the singularity. 
A related and highly analyzed isolated point of nonuniqueness is given by the RayleighTaylor instability. A planar surface separates an incompressible heavy fluid (above) from a light fluid (below). This initial condition is an isolated unstable point in the space of initial conditions, in that small perturbations will diverge from it, with the solution depending on the perturbation. The Rayleigh-Taylor initial conditions are statically unstable, while the Taylor-Green vortex is dynamically unstable, with its degree of instability diverging as the critical time approaches.

While the Rayleigh-Taylor static instability point does not require any reformulation of the uniqueness concepts of solutions, the Taylor-Green isolated point of dynamic instability should be considered in any formulation of uniqueness for the solutions of the Euler equations.

Nonuniqueness of solutions of the Euler equations has been established mathematically for incompressible flow in [11, 12, 45], and for compressible flow in [5, 6, 24] and the references cited therein. These solutions are very sparse in their $k$ space representation, and are far removed from any type of energy cascade and scaling law which could be associated with the K41 turbulence or its corrections. From the perspective of fundamental physics, the physical solution should be the one that maximizes the rate of dissipation of entropy among all possible solutions of the Euler equations. We emphasize that entropy has the familiar thermodynamic aspect concerning molecular motions (i.e., heat released), but it also includes turbulent fluid fluctuations. Such a condition is inconvenient to apply and leaves open the statement of a convenient entropy principle. As indicated earlier, for the incompressible Euler equations, (CKHi) implies constant energy in time for the solution ( $c f$. [4]). For the barotropic compressible Euler equations, the energy cannot be constant as it is dissipated (decreases) within a shock wave. Thus, we propose as a necessary admissibility condition for physically meaningful solutions of the Euler equations that they should be a strong limit of the Navier-Stokes solutions with a uniform bound (CKHi). To our knowledge, the wild solutions do not satisfy either of these conditions. We note that intermittency bounds as in (CKHi) are insufficient to force maximization of the entropy decay rate, due to higher order intermittency structures contributing to the entropy. We do not know of a convenient reformulation of this condition.

At a deeper level, we comment on difficulties with the principle of maximum rate of entropy production as a strengthened statement of the second law of thermodynamics. Assessment of this principle for the purpose of validation or verification depends on solutions of a variational principle. The variational principles for conservative equations $(\delta \mathcal{L}=0$, where $\mathcal{L}$ is the system Lagrangian) and for dissipative systems (an entropy rate condition) are known, but a gap in the formulation of mechanics is the absence of a variational formulation for systems that combine conservative and dissipative components.

The numerical simulation model called Implicit Large Eddy Simulation (ILES) for turbulent flows has a sizable following, although controversial within the larger turbulent simulation community. By design, it is located exactly at the marginal decay rate $-\frac{5}{3}$, and further, by design, the associated prefactor is minimized, so that it is smaller than $\epsilon^{\frac{2}{3}}$. In an analysis to be published separately, we will present reasons for believing that the ILES solutions fail to maximize the entropy dissipation rate among the competing (nonunique) solutions of the Euler equations, or in other words, that ILES are not physically meaningful.

Acknowledgments. The research of Gui-Qiang G. Chen was supported in part by the UK Engineering and Physical Sciences Research Council Award EP/E035027/1 and 
EP/L015811/1, and the Royal Society-Wolfson Research Merit Award (UK). James Glimm's research was supported in part by Stanford University (PSAAP/DOE) funding.

\section{REFERENCES}

[1] G. Batchelor, The Theory of Homogeneous Turbulence, Cambridge University Press: Cambridge, 1953.

[2] M. E. Brachet, Direct simulation of three dimensional turbulence in the Taylor-Green vortex, Fluid Dynamics Research, 8 (1991), 1-8.

[3] G.-Q. Chen and J. Glimm, Kolmogorov's theory of turbulence and inviscid limit of the Navier-Stokes equations in $\mathbb{R}^{3}$, Commun. Math. Phys. 310 (2012), 267-283.

[4] A Cheskidov, P Constantin, S Friedlander, and R. Shvydkoy, Energy conservation and Onsagers conjecture for the Euler equations, Nonlinearity, 21 (2008), 1233-1252.

[5] E. Chiodaroli, C. De Lellis, and O. Kreml, Global ill-posedness of the isentropic system of gas dynamics, Comm. Pure Appl. Math. 68 (2015), 1157-1190.

[6] E. Chiodaroli and O. Kreml, Non-uniqueness of admissible weak solutions to the Riemann problem for the isentropic Euler equations, Nonlinearity, 31 (2018), 1441-1460.

[7] P. Constantin, Navier-Stokes equations and area of interfaces, Commun. Math. Phys. 129 (1990), 241266.

[8] P. Constantin, On the Euler equations of incompressible fluids, Bull. Amer. Math. Soc. 44 (2007), 603621.

[9] P. Constantin and C. Doering, Variational bounds on dissipative systems, Physica D 82 (1995), 221228; Variational bounds on energy dissipation in incompressible flows II. Channel flow, Phys. Rev. E 51 (1995), 3192-3198.

[10] J. R. DeBonis, Solutions of the Taylor-Green vortex problem using high-resolution explicit finite difference methods, In: Conference Procceedings of the 51st AIAA Aerospace Sciences Meeting including the New Horizons Forum and Aerospace Exposition, 7-10 January 2013, Grapevine (Dallas/Ft. Worth Region), Texas, AIAA 2013-0382, https://arc.aiaa.org/doi/10.2514/6.2013-382.

[11] C. De Leliss and L. Székelyhidi Jr. The Euler equations as a differential inclusion. Ann. Math. (2), 170 (2009), 1417-1436.

[12] C. De Leliss and L. Székelyhidi Jr. On admissibility criteria for weak solutions of the Euler equations. Arch. Ration. Mech. Anal. 195 (2010), 225-260.

[13] C. R. Doering and C. Foias, Energy dissipation in body-forced turbulence, J. Fluid Mech. 467 (2002), 289-306.

[14] C. Federrath, On the universality of supersonic turbulence, Mon. Not. R. Astron. Soc. 436(2) (2013), 1245-1257.

[15] E. Feireisl, T. Karper, and M. Pokorny, Mathematical Theory of Compressible Viscous Fluids: Analysis and Numerics. Lecture Notes in Mathematical Fluid Mechanics. Birkhäuser/Springer, Cham, 2016.

[16] E. Feireisl, A. Novotny, and H. Petzeltov, On the existence of globally defined weak solutions to the NavierCStokes equations, J. Math. Fluid Mech. 3 (2001), 358-392.

[17] E. Feireisl, Dynamics of Viscous Compressible Fluids. Oxford University Press: Oxford, 2004.

[18] C. Foias, O. Manley, R. Rosa, and R. Temam, Navier-Stokes Equations and Turbulence, Cambridge University Press: Cambridge, 2001.

[19] U. Frisch, Turbulence: The Legacy of A. N. Kolmogorov, Cambridge Univeristy Press: Cambridge, 1996.

[20] U. Frisch, T. Matsumoto, and J. Bec, Singularities of Euler flow? Not out of the blue! J. Stat. Phys. 113 (2003), 761-781.

[21] W. Gangbo and M. Westdickenberg, Optimal transport for the system of isentropic Euler equations. Comm. Partial Diff. Eqs. 34 (2009), 1041-1073.

[22] J. Glimm and V. Mahadeo, A scaling law and closed form K62 turbulent statistics, Preprint, 2018.

[23] E. Hopf, Über die Anfangswertaufgabe für die hydrodynamischen Grundgleichungen. Math. Nachr. 4 (1951), 213-231.

[24] C. Klingenberg and S. Markfelder, The Riemann problem for the multidimensional isentropic system of gas dynamics is ill-posed if it contains a shock, Arch. Ration. Mech. Anal. 227 (227), 967-994.

[25] A. N. Kolmogoroff, The local structure of turbulence in incompressible viscous fluid for very large Reynold's numbers. C. R. (Doklady) Acad. Sci. URSS (N.S.) 30 (1941), 301-305. 
[26] A. N. Kolmogoroff, Dissipation of energy in the locally isotropic turbulence. C. R. (Doklady) Acad. Sci. URSS (N.S.), 32 (1941), 16-18.

[27] A. N. Kolmogoroff, A refinement of previous hypotheses concerning the local structure of turbulence in a viscous incompressible fluid at high Reynolds number, J. Fluid Mech. 13 (1962), 82-85.

[28] A. G. Kritsuk, S. D. Ustyugov, M. L. Norman, and P. Padoan, Self-organization in turbulent molecular clouds: compressional versus solenoidal modes, In: Numerical Modeling of Space Plasma Flows, Astronum-2009, ed. by N.V. Pogorelov, E. Audit, G.P. Zank. Astronomical Society of the Pacific Conference Series, Vol. 429, 2010.

[29] J. Leray, Etude de diverses équations intégrales nonlinéaires et de queques problémes que pose l'hydrodynamique. J. Math. Pures Appl. 12 (1933), 1-82.

[30] J. Leray, Essai sur les mouvements plans d'un liquide visqueux que limitent des parois. J. Math. Pures Appl. 13 (1934), 331-418.

[31] J. Leray, Sur le mouvement d'un liquide visqueux emplissant l'espace. Acta Math. 63 (1934), $193-248$.

[32] Y. Li, E. Perlman, M. Wan, Y. Yang, R. Burns, C. Meneveau, R. Burns, S. Chen, A. Szalay, and G. Eyink, A public turbulence database cluster and applications to study Lagrangian evolution of velocity increments in turbulence, J. Turbulence, 9, no. 31, 2008.

[33] H. Lim, Y. Yu, J. Glimm, X.-L. Li, and D. H. Sharp, Chaos, transport, and mesh convergence for fluid mixing, Acta Math. Appl. Sinica 24 (2008), 355-368.

[34] H. Lim, Y. Yu, J. Glimm, X. L. Li, and D. H. Sharp, Subgrid models for mass and thermal diffusion in turbulent mixing, Physica Scripta, T142 (2010), 014062.

[35] J.-L. Lions, Quelques Méthodes de Résolution des Problémes aux Limites Nonlinea'eaires. Dunod, Paris, 1969.

[36] P.-L. Lions, Mathematical Topics in Fluid Mechanics. Vol. 1: Incompressible Models, Oxford: Oxford Sci. Pub., 1996.

[37] A. J. Majda and A. L. Bertozzi, Vorticity and Incomprerssible Flow, Cambridge University Press: Cambridge, 2001.

[38] W. D. McComb, The Physics of Fluid Turbulence, Oxford University Press: Oxford, 1990.

[39] A. S. Monin and A. M. Yaglom, Statistical Fluid Mechanics: Mechanics of Turbulence, Vol. I-II. Translated from the 1965 Russian original. Edited and with a preface by John L. Lumley. English edition updated, augmented and revised by the authors. Dover Publications, Inc., Mineola, NY, 2007.

[40] J. Neustupa, Measure-valued solutions of the Euler and Navier-Stokes equations for compressible barotropic fluids, Math. Nachr. 163 (1993), 217-227.

[41] A. Matsumura and T. Nishida, The initial value problem for the equations of motion of viscous and heat-conductive gases, J. Math. Kyoto Univ. 20 (1980), 67-104.

[42] A. M. Oboukhov, Some specific features of atmospheric tubulence, J. Fluid Mech. 13 (1962), 77-81.

[43] L. Onsager, Statisitical Hydrodynamics, Nuovo Cimento (Suppl.), 6 (1949), 279-287.

[44] E. Perlman, R. Burns, Y. Li, and C. Meneveau, Data exploration of turbulence simulations using a database cluster, In: Proceedings of the 2007 ACM/IEEE Conference on Supercomputing, Article No. 23, Reno, Nevada, November 10-16, 2007.

[45] V. Scheffer, An inviscid flow with compact support in space-time, J. Geom. Anal. 3 (1993), $343-401$.

[46] Bohua Sun, Scaling laws for compressible turbulence, Appl. Math. Mech. 38 (2017), 765-778.

[47] L. Tartar, Compensated compactness and applications to partial differential equations, Research Notes in Mathematics, Nonlinear Analysis and Mechanics, Herriot-Watt Symposium, Vol. 4, Knops R.J. ed., Pitman Press, 1979.

[48] R. Temam, Navier-Stokes Equations, North-Holland, Amsterdam, 1977.

[49] J.-C. Wang, Y. Yang, Y. Shi, Z. Xian, and S. Chen, Cascade of kinetic energy in three-dimensional compressible turbulence, Phys. Rev. Lett. 110 (2013), 214505.

[50] H. Ziegler, An Introduction to Thermomechanics, North Holland, 1987.

Gui-Qiang G. Chen, Mathematical Institute, University of Oxford, Oxford, OX2 6GG, UK

E-mail address: chengq@maths.ox.ac.uk

James Glimm, Department of Applied Mathematics and Statistics, Stony Brook University, Stony Brook, NY 11794-3600, USA

E-mail address: glimm@ams.sunysb.edu 